\title{
Data report: elemental, Rock-Eval, and isotopic compositions of bulk sediments, IODP Expedition $311^{1}$
}

\author{
Ji-Hoon Kim² and Young-Joo Lee ${ }^{2}$
}

\section{Chapter contents}

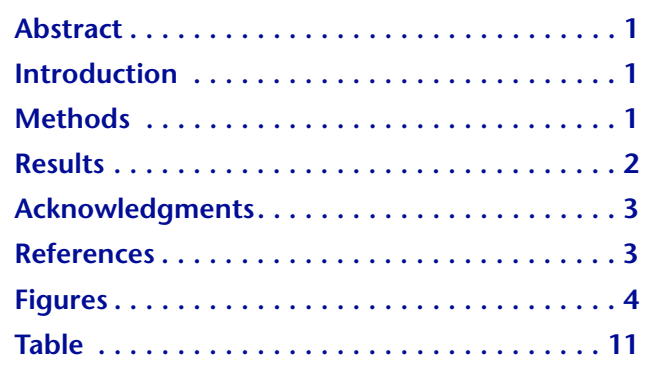

${ }^{1}$ Kim, J.-H., and Lee, Y.-J., 2009. Data report: elemental, Rock-Eval, and isotopic compositions of bulk sediments, IODP Expedition 311. In Riedel, M., Collett, T.S., Malone, M.J., and the Expedition 311 Scientists, Proc. IODP, 311: Washington, DC (Integrated Ocean Drilling Program Management International, Inc.).

doi:10.2204/iodp.proc.311.207.2009

${ }^{2}$ Petroleum and Marine Resources Research

Division, Korean Institute of Geoscience and

Mineral Resources, 30 Gajeong-dong, Yuseong-gu, Daejeon 305-350, Korea.

Correspondence author: save@kigam.re.kr

\section{Abstract}

Several geochemical analyses were performed on samples to assess their geochemical characteristics and variations according to vertical depth and site location. Total carbon, total organic carbon (TOC), and total nitrogen (TN) content decreased from northeast to southwest along the transect of Integrated Ocean Drilling Program Expedition 311 Sites U1329, U1327, U1325, and U1326. Variations with depth showed differing trends with sites and compositions. Most organic matter was at an immature stage and Type III evolution based on Rock-Eval pyrolysis. However, results from TOC/TN, $\delta^{13} \mathrm{C}_{\text {org }}$, and $\delta^{15} \mathrm{~N}_{\text {org }}$ analyses showed that organic matter has an admixture of origin.

\section{Introduction}

Operations during Integrated Ocean Drilling Program (IODP) Expedition 311 drilled five sites across the northern Cascadia margin (see the "Expedition 311 summary" chapter). Among the five sites, the northeast-southwest transect of Sites U1329, U1327, U1325, and U1326 was cored to investigate gas hydrate occurrence and formation. Cold vent Site U1328 was also drilled. Based on recovered core sections, Sites U1329, U1327, U1326, and U1328 are divided into three lithostratigraphic units (I, II, and III) and Site U1325 is divided into five lithostratigraphic subunits (IA, IB, II, III, and IV) (see the "Expedition 311 summary" chapter). Characterization of elements (total carbon [TC], total nitrogen [TN], and total sulfur [TS]) and organic matter in bulk sediments according to transect and lithostratigraphic units could be helpful in understanding the depositional environment, diagenesis, and origin of organic matter and give additional information about gas hydrate occurrence. Hence, this study reports several geochemical results on bulk sediments from the northern Cascadia margin.

\section{Methods \\ Elemental analysis}

A total of 311 squeeze cake samples from Sites U1325-U1329 were used for geochemical analyses. A part of each squeeze cake was dried for $24 \mathrm{~h}$ using a freeze dryer at the Korea Institute of Geoscience and Mineral Resources. After drying the bulk sediment sam- 
ples were ground and homogenized in an agate mortar. TC and TN contents were measured by combustion method using a LECO CHN-900 apparatus with detection limits of $0.001 \%$ for TC and $0.01 \%$ for TN. TS contents were determined using a LECO SC-132 apparatus with a detection limit of $0.001 \%$.

\section{Rock-Eval pyrolysis}

Using Rock-Eval pyrolysis, free and adsorbed hydrocarbons released during programmed heating of a sample are recorded as the first peak in a pyrogram $\left(S_{1}\right)$ under low temperature. The second peak $\left(S_{2}\right)$ in the pyrogram represents hydrocarbons released by kerogen cracking when the sample is heated to $550^{\circ} \mathrm{C}$. The temperature at the maximum of the $S_{2}$ peak is defined as $T_{\max }$. $\mathrm{CO}_{2}$, shown as the third peak $\left(\mathrm{S}_{3}\right)$ in the program, is also generated by kerogen degradation. When these components are normalized to the TOC content, the $S_{2}$ peak becomes the hydrogen index $\left(\mathrm{HI}=\mathrm{S}_{2} \times 100 / \mathrm{TOC}\right)$ and $\mathrm{S}_{3}$ becomes the oxygen index (OI $\left.=\mathrm{S}_{3} \times 100 / \mathrm{TOC}\right)$ (Tissot and Welte, 1984; Peters, 1986). Rock-Eval pyrolysis and TOC were determined by Rock-Eval 6.

\section{Isotope analysis}

Powdered bulk samples were pretreated with $3 \mathrm{~N} \mathrm{HCl}$ to remove carbonate $\left(\mathrm{CaCO}_{3}\right)$ and inorganic nitrogen for the analysis of organic carbon $\left(\delta^{13} \mathrm{C}_{\text {org }}\right)$ and nitrogen $\left(\delta^{15} \mathrm{~N}_{\text {org }}\right)$ isotope ratios. $\delta^{13} \mathrm{C}_{\text {org }}$ and $\delta^{15} \mathrm{~N}_{\text {org }}$ were measured by a VG Optima stable isotope ratio mass spectrometer at the National Instrumentation Center for Environmental Management. The analytical reproducibility was $\pm 0.1 \%$ for $\delta^{13} \mathrm{C}_{\text {org }}$ and $\pm 0.2 \% 0$ for $\delta^{15} \mathrm{~N}_{\text {org }}$. All carbon and nitrogen isotopes are reported in the usual $\delta$ notation relative to Vienna Peedee belemnite (VPDB) for carbon and atmospheric $\mathrm{N}_{2}$ for nitrogen:

$$
\delta(\% 0)=\left(\frac{R_{\text {sample }}-R_{\text {Standard }}}{R_{\text {standard }}}\right) \times 1000,
$$

where $R$ represents the ${ }^{13} \mathrm{C} /{ }^{12} \mathrm{C}$ ratio and ${ }^{15} \mathrm{~N} /{ }^{14} \mathrm{~N}$ ratio of the sample and standard for each isotope.

\section{Results}

\section{Elemental composition}

TC, TOC, and TN contents show spatial variation across the transect of four sites (Table T1; Figs. F1, F2). Their values gradually decrease from land toward open sea. That is, the highest values are at Site U1329 and the lowest values are at Site U1326. Variation with depth show a similar trend among these el- ements. TC, TOC, and TN values slightly increase with depth at Sites U1329 and U1327, whereas they decrease with depth at Sites U1325 and U1326 (Fig. F1). In particular, TC values reach $7.34 \mathrm{wt} \%$ in the upper part of Site U1328 (Fig. F1), where many massive gas hydrates and authigenic carbonates were found on board (see the "Site U1328" chapter).

TOC and TN show a strong positive correlation $\left(R^{2}>\right.$ 0.80) (Table T1). Also, moderate or strong positive correlations were observed between TC and TOC and between TC and TN, except for an abnormal value.

Organic matter from marine algae typically has an atomic TOC/TN ratio of 4-10; ratios from vascular land plants are $\geq 20$ (Emerson and Hedges, 1988; Meyers, 1994). Most atomic TOC/TN ratios in tested Expedition 311 samples have a range of 4-10 (Table T1; Figs. F1, F2) and are relatively constant with depth.

TS content at Site U1328 is higher at the surface and shows a slightly increasing trend with depth. At Site U1326, TS content slightly decreases with depth (Fig. F1). TOC/TS ratios at Sites U1326 and U1328 have a slightly decreasing trend with depth, whereas they are slightly increasing at Sites U1325 and U1329 (Fig. F1).

\section{Rock-Eval pyrolysis}

Most analyzed $S_{2}$ and $S_{3}$ values from Expedition 311 samples are $<3 \mathrm{mg}$ hydrocarbon $[\mathrm{HC}] / \mathrm{g}$ rock and 3 $\mathrm{mg} \mathrm{CO}_{2} / \mathrm{g}$ rock (Table T1; Fig. F3). $\mathrm{S}_{2}$ values show vertical and spatial variations through the transect, whereas $\mathrm{S}_{3}$ values do not show any trend (Fig. F3). $\mathrm{S}_{2}$ values are higher at Sites U1329 and U1327, located nearer to land, than at other sites. Additionally, $\mathrm{S}_{2}$ values at Sites U1329 and U1327 slightly increase with depth, whereas $S_{2}$ values at the other sites are relatively constant irrespective of depth (Fig. F3).

Most analyzed $\mathrm{HI}$ and $\mathrm{OI}$ values are $50-150 \mathrm{mg} \mathrm{HC} / \mathrm{g}$ TOC and 100-300 $\mathrm{mg} \mathrm{CO}_{2}$ /g TOC (Table T1; Figs. F3, F4). Plotting modified van Krevelen-type and $\mathrm{S}_{2}$ versus TOC diagrams using analyzed values from Expedition 311, most samples are at Type III evolution. There is a strong positive correlation $\left(R^{2}>0.80\right)$ between $\mathrm{S}_{2}$ and TOC (Fig. F4).

Most analyzed $T_{\max }$ values were $<435^{\circ} \mathrm{C}$, which shows that organic matter in the samples is at a thermally immature stage. $T_{\max }$ did not show vertical variation with depth (Table T1; Fig. F3). However, $T_{\max }$ has the lowest values $\left(<350^{\circ} \mathrm{C}\right)$ in the upper part of lithostratigraphic Unit I $(<50$ meters below seafloor) at Site U1328 (Fig. F3). This interval contains massive in situ gas hydrates (see the "Site U1328" chapter). Additionally, the $T_{\max }$ of Unit III at 
Site U1326 has a lower value and shows variation (Fig. F3).

\section{Isotopic composition}

Marine organic matter typically has $\delta^{13} \mathrm{C}$ values from $-22 \%$ to $-20 \%$ (Jasper and Gagosian, 1990; Meyers, 1994). Measured $\delta^{13} \mathrm{C}_{\text {org }}$ values of Expedition 311 samples generally are from $-27.5 \%$ to $-22.5 \%$. $\delta^{13} \mathrm{C}_{\text {org }}$ values are higher at Sites U1329 and U1327 compared to the other sites (Table T1; Figs. F1, F2). $\delta^{13} \mathrm{C}_{\text {org }}$ values slightly increase with depth at Sites U1329, U1327, and U1328, whereas they show a slightly decreasing tend at Site U1325 (Figs. F1, F2).

Analyzed $\delta^{15} \mathrm{~N}_{\text {org }}$ values are from $-3 \%$ to $6 \%$. $\delta^{15} \mathrm{~N}_{\text {org }}$ values are higher at Sites U1329, U1327, and U1328 than at Sites U1325 and U1326. $\delta^{15} \mathrm{~N}_{\text {org }}$ values slightly decrease with depth at Sites U1329, U1327, and U1325, whereas they show a slightly increasing tend at Site U1328 (Table T1; Figs. F1, F2).

\section{Acknowledgments}

This study used samples provided by the Integrated Ocean Drilling Program (IODP) and was financially supported by Korea Integrated Ocean Drilling Program (K-IODP) of Ministry of Land, Transport and Maritime Affairs (MLTM).

\section{References}

Emerson, S., and Hedges, J.I., 1988. Processes controlling the organic carbon content of open ocean sediments. Paleoceanography, 3:621-634.

Jasper, J.P., and Gagosian, R.B., 1990. The sources and deposition of organic matter in the late Quaternary Pigmy Basin, Gulf of Mexico. Geochim. Cosmochim. Acta, 54(4):1117-1132. doi:10.1016/0016-7037(90)90443-O

Meyers, P.A., 1994. Preservation of elemental and isotopic source identification of sedimentary organic matter. Chem. Geol., 144:289-302. doi:10.1016/ 0009-2541(94)90059-0

Peters, K.E., 1986. Guidelines for evaluating petroleum source rock using programmed pyrolysis. AAPG Bull., 70:318-329.

Tissot, B.P., and Welte, D.H., 1984. Petroleum Formation and Occurrence (2nd ed.): Heidelberg (Springer-Verlag).

Initial receipt: 25 June 2008

Acceptance: 27 October 2008

Publication: 8 May 2009

MS 311-207 
Figure F1. Element concentrations, ratios, and isotope profiles. TC $=$ total carbon, TOC $=$ total organic carbon, $\mathrm{TN}=$ total nitrogen, TS = total sulfur. A. Site U1325. B. Site U1326. C. Site U1327. (Continued on next page.)

A $\quad$ TC (wt\%) $\quad$ TC (wt\%) $\quad$ TN (wt\%) $\quad$ TS (wt\%) $\quad$ TOC/TN $\quad T O C / T S \quad \delta^{13} C_{\text {org }}(\%) \quad \delta^{15} \mathrm{~N}_{\text {org }}(\%)$
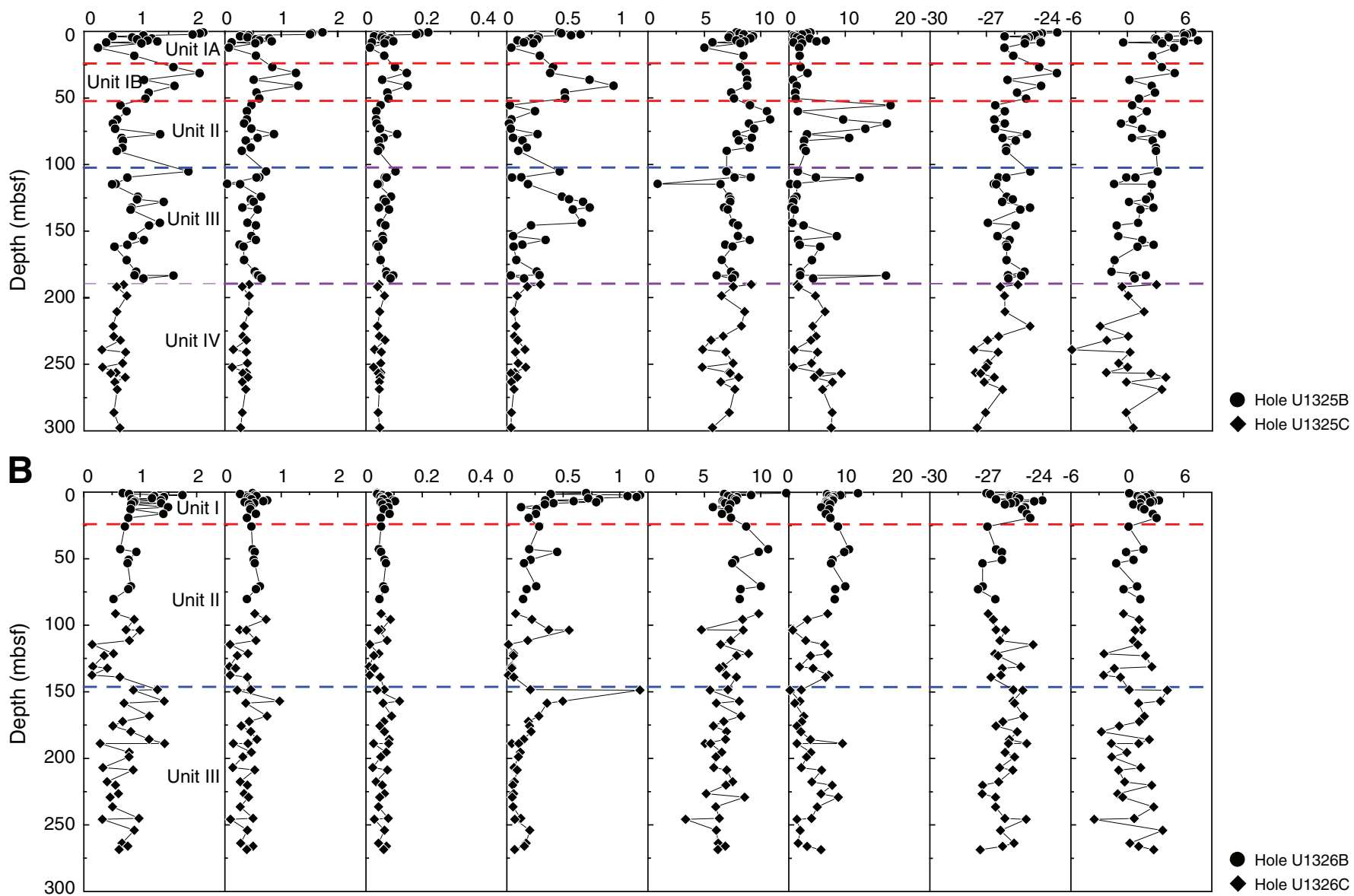

- Hole U1326B

C

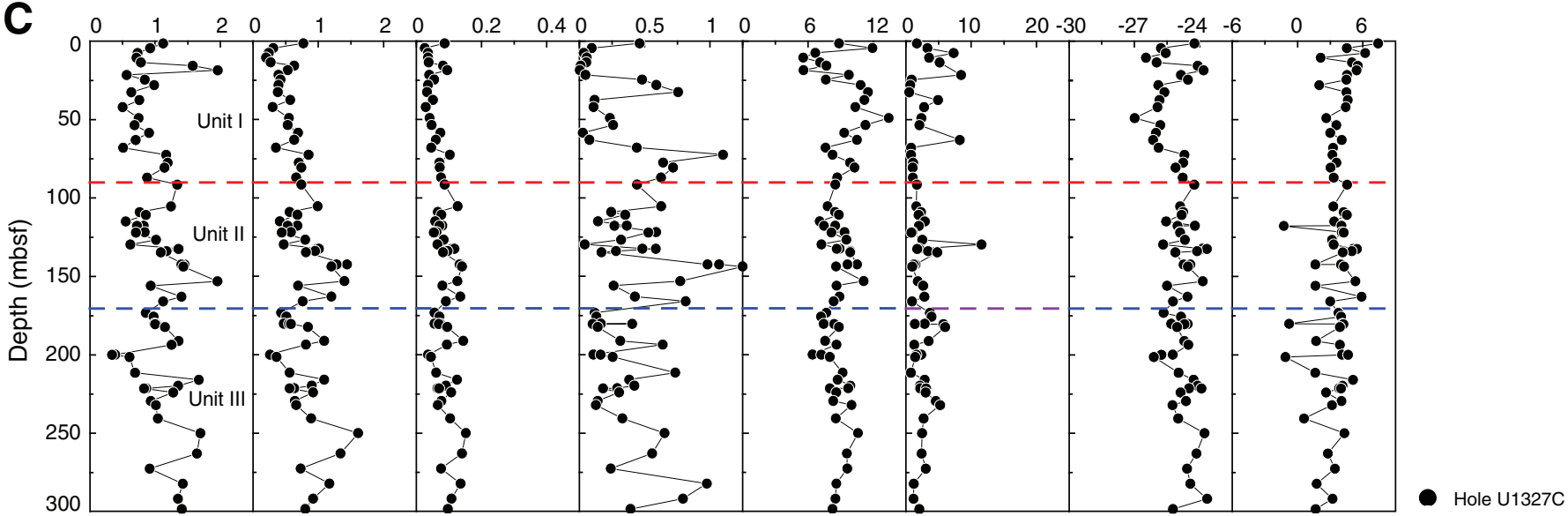


Figure F1 (continued). D. Site U1328. E. Site U1329.

D TC (wt\%) TC (wt\%) $\quad$ TN (wt\%) TS (wt\%) $\quad$ TOC/TN $\quad T O C / T S \quad \delta^{13} \mathrm{C}_{\text {org }}(\%) \quad \delta^{15} \mathrm{~N}_{\text {org }}(\%)$

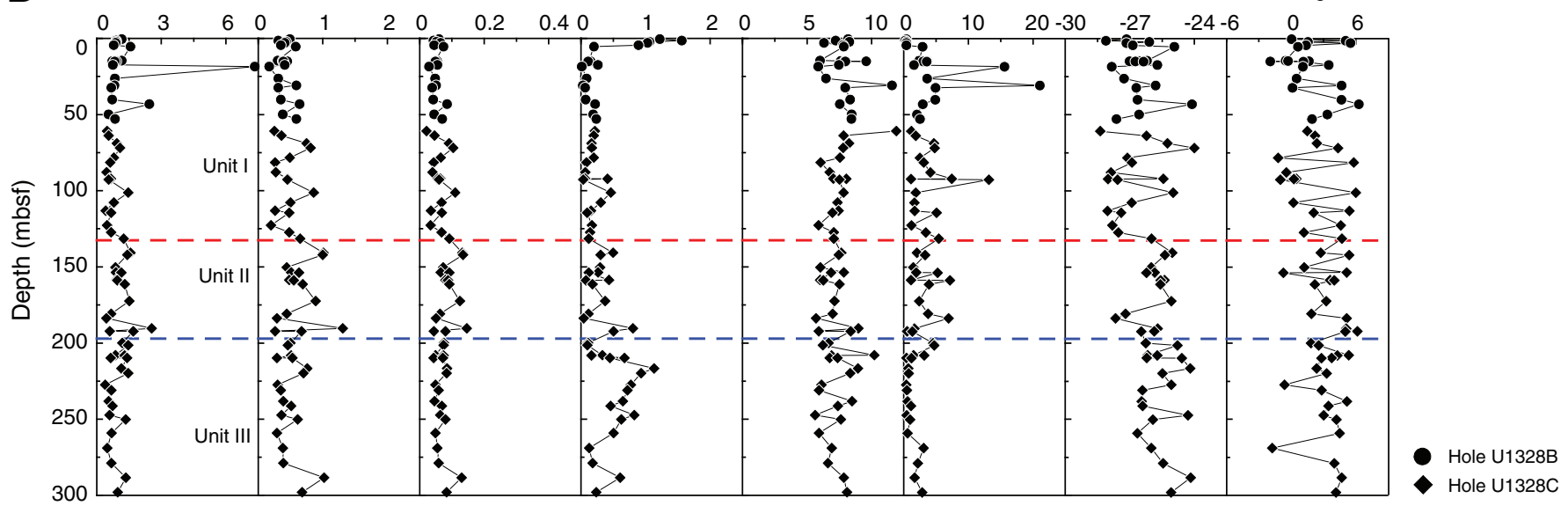

E

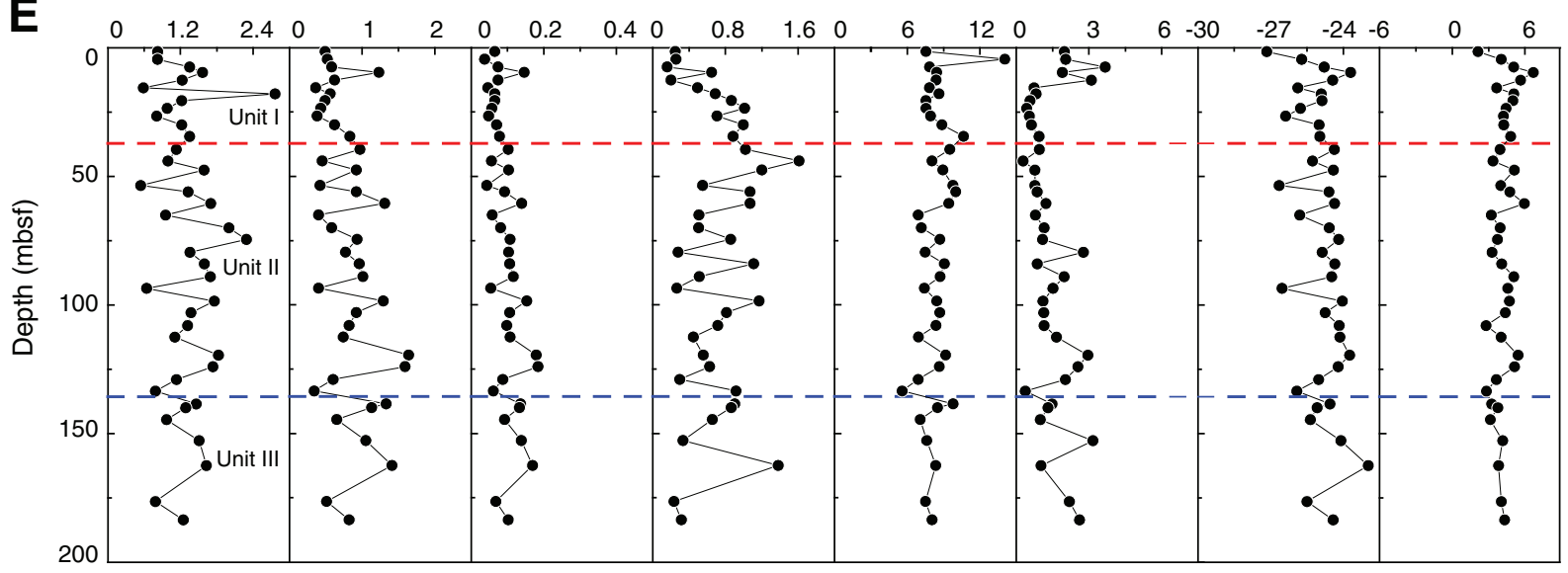

Hole U1329C 
Figure F2. Element concentration, ratio, and isotope values by lithostratigraphic unit. Horizontal line = median, bottom box $=$ first quartile $\left(\mathrm{Q}_{1}\right)$, top box $=$ third quartile $\left(\mathrm{Q}_{3}\right)$, whiskers = lowest and highest observations inside the regions $\mathrm{Q}_{1}-1.5\left(\mathrm{Q}_{3}-\mathrm{Q}_{1}\right)$ and $\mathrm{Q}_{3}-1.5\left(\mathrm{Q}_{3}-\mathrm{Q}_{1}\right)$, respectively. ${ }^{*}=$ outliers. $\mathrm{A}$. Total carbon (TC). B. Total organic carbon (TOC). C. Total nitrogen (TN). D. Total sulfur (TS). E. TOC/TN. F. TOC/TS. G. Carbon isotope. H. Nitrogen isotope.

A

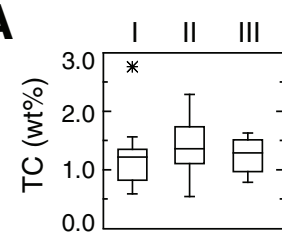

B

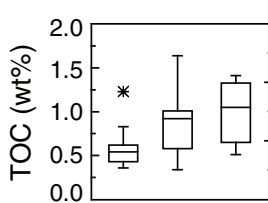

C

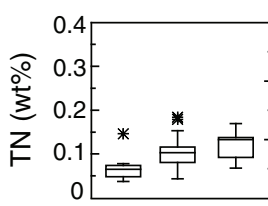

D

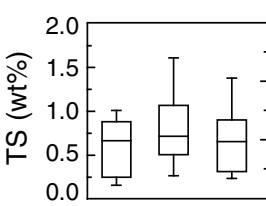

E

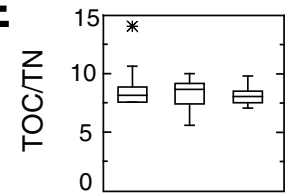

$\mathbf{F}$
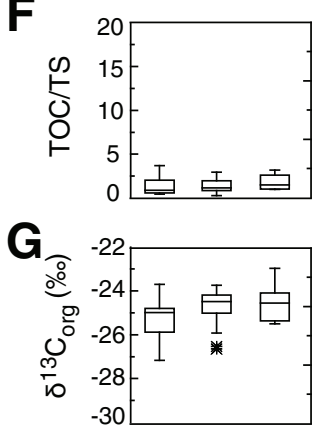

H

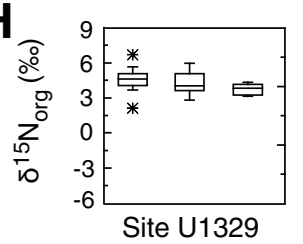

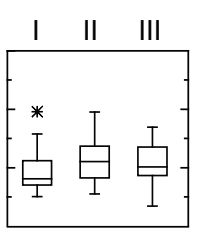
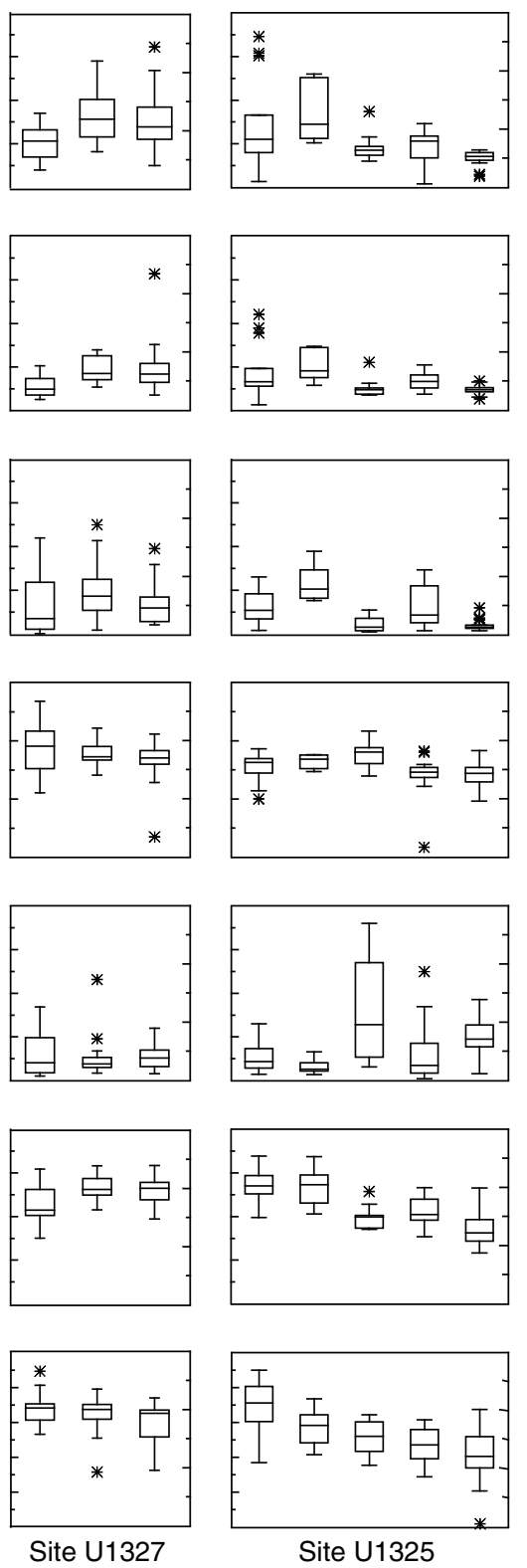
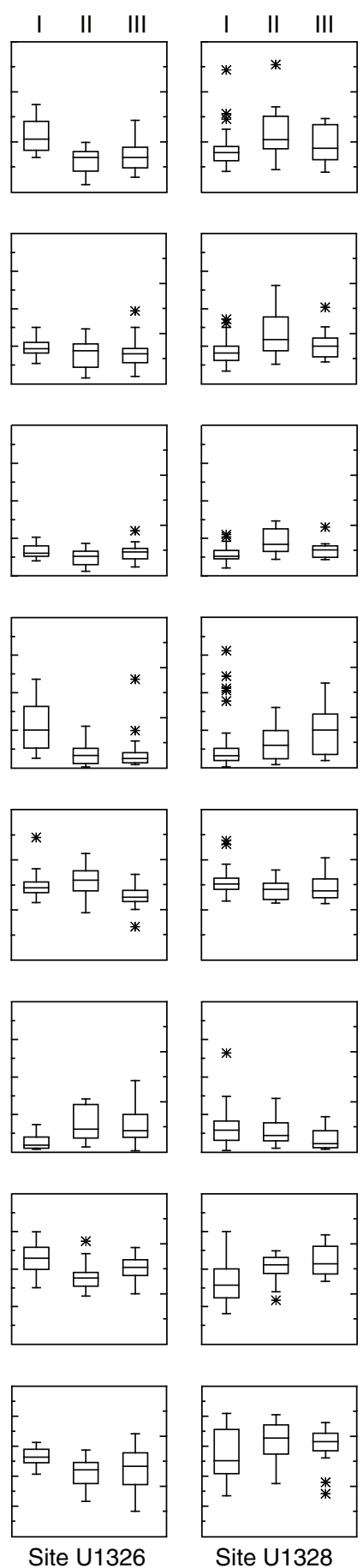
Figure F3. Rock-Eval pyrolysis profiles. $S_{2}=$ second peak, $S_{3}=$ third peak, $T_{\max }=$ temperature at $S_{2}, H I=$ hydrogen index, $\mathrm{HC}=$ hydrocarbon, TOC $=$ total organic carbon, OI = oxygen index. A. Site U1325. B. Site U1326. C. Site U1327. (Continued on next page.)
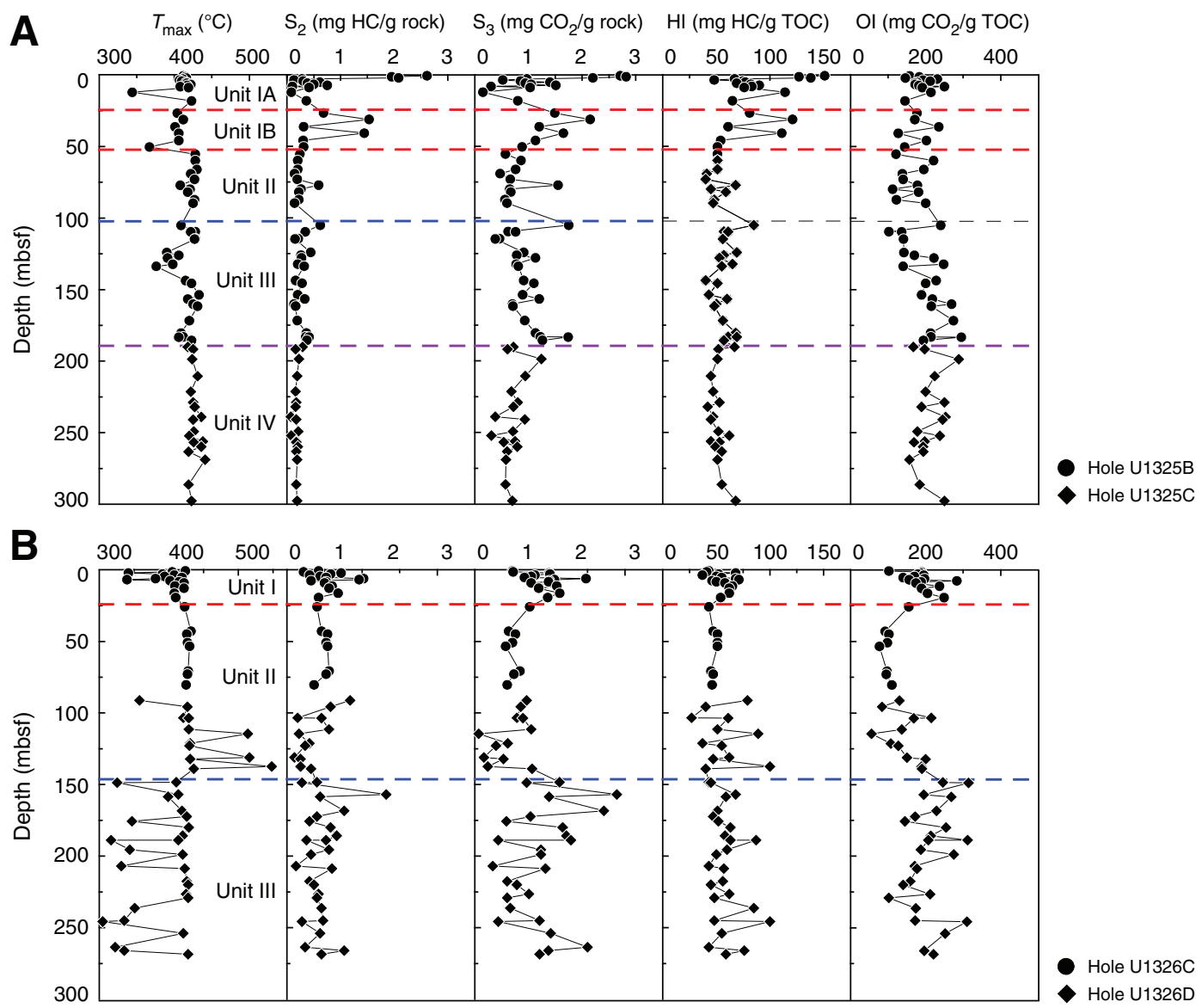

Hole U1326C C $\quad \begin{array}{llllllllllllllllll}300 & 400 & 500 & 0 & 1 & 2 & 3 & 0 & 1 & 2 & 3 & 0 & 50 & 100 & 150 & 0 & 200 & 400\end{array}$

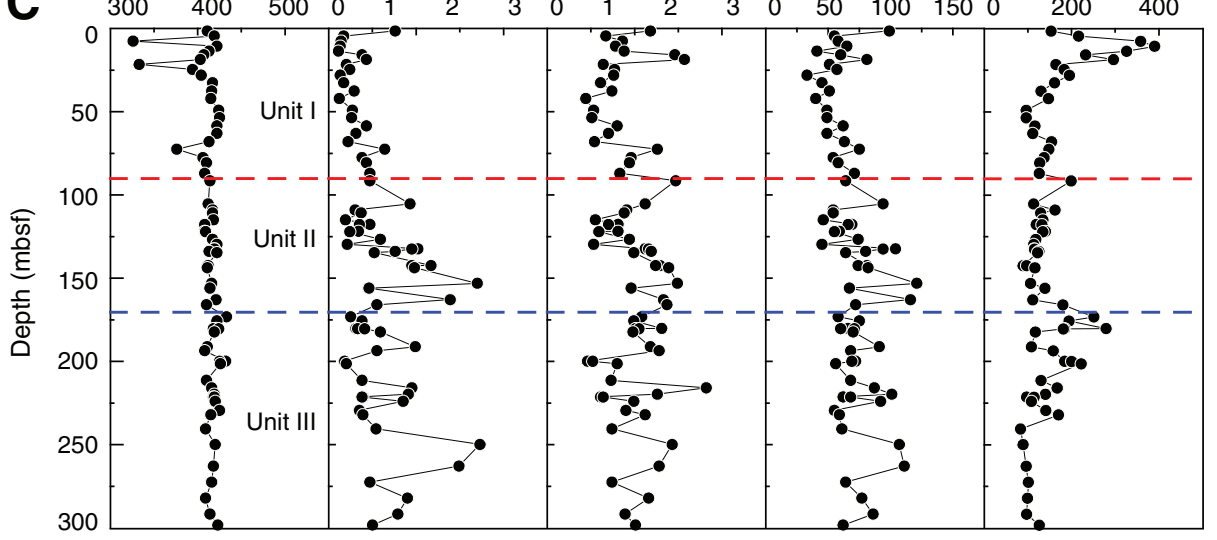

Hole U1327C 
Figure F3 (continued). D. Site U1328. E. Site U1329.
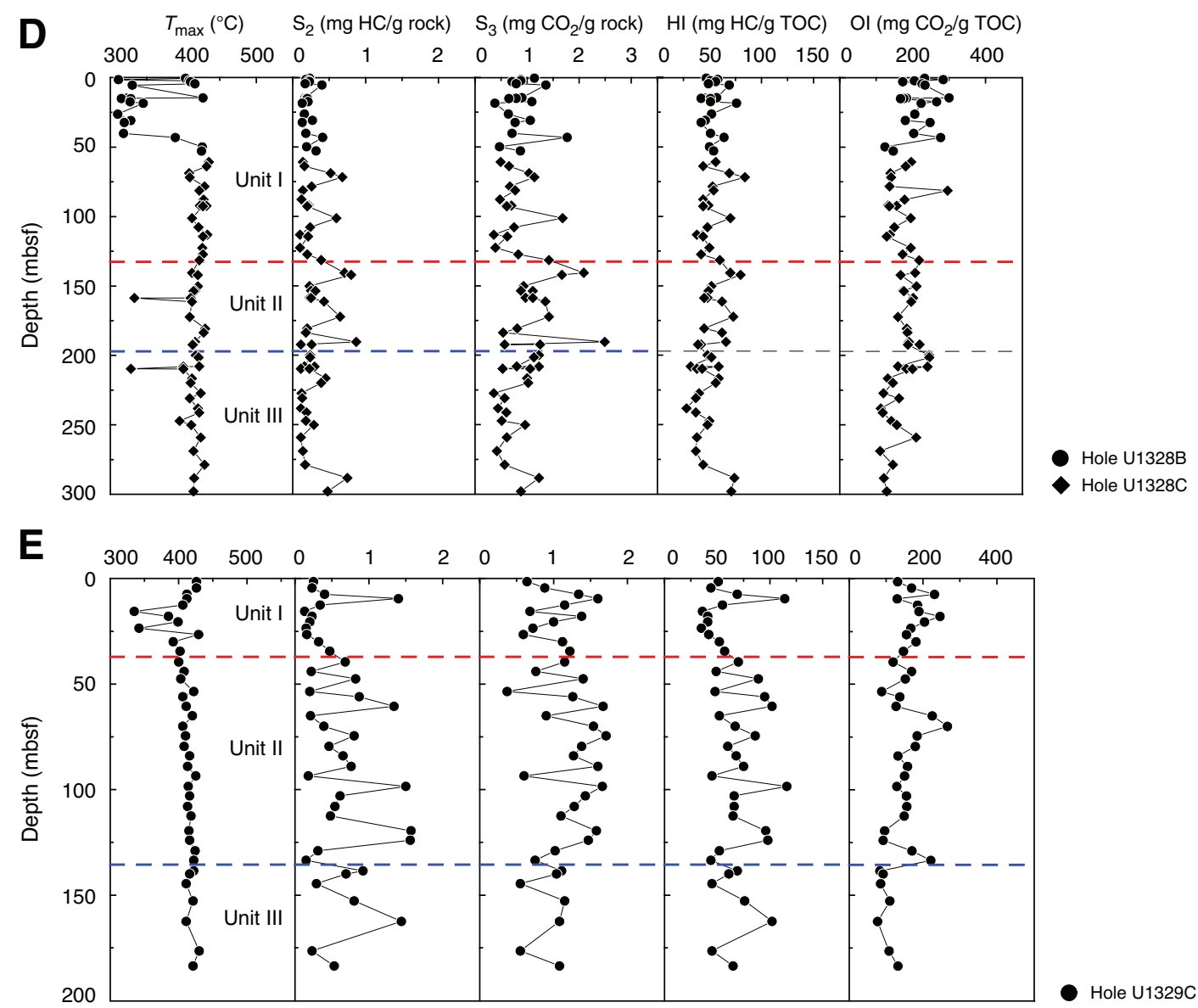
Figure F4. Modified van Krevelen-type diagrams and cross-plots. HI = hydrogen index, HC = hydrocarbon, OI $=$ oxygen index, TOC $=$ total organic carbon. A. Site U1325. B. Site U1326. C. Site U1327. (Continued on next page.)
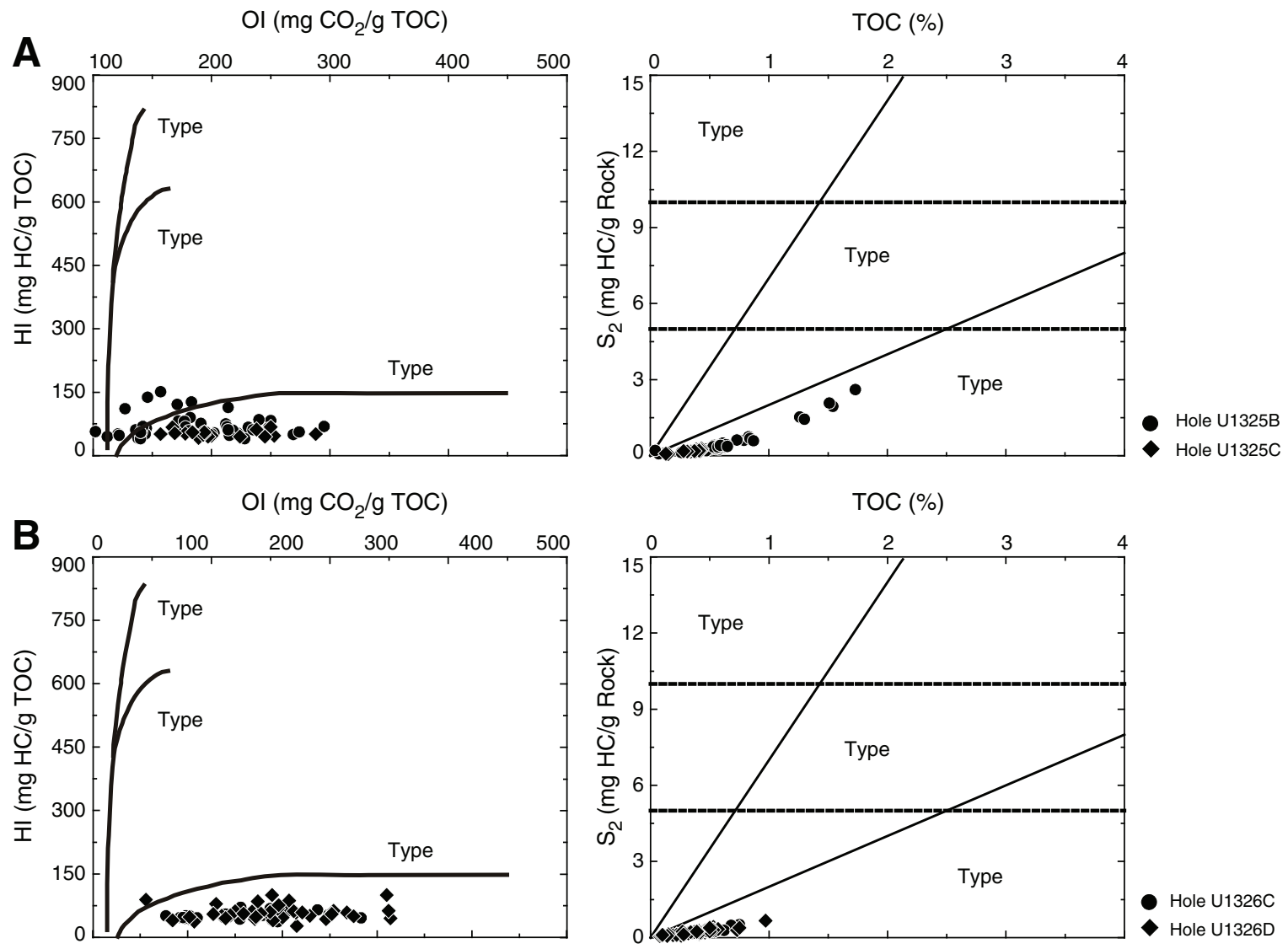

$\mathrm{Ol}\left(\mathrm{mg} \mathrm{CO}_{2} / \mathrm{g}\right.$ TOC)
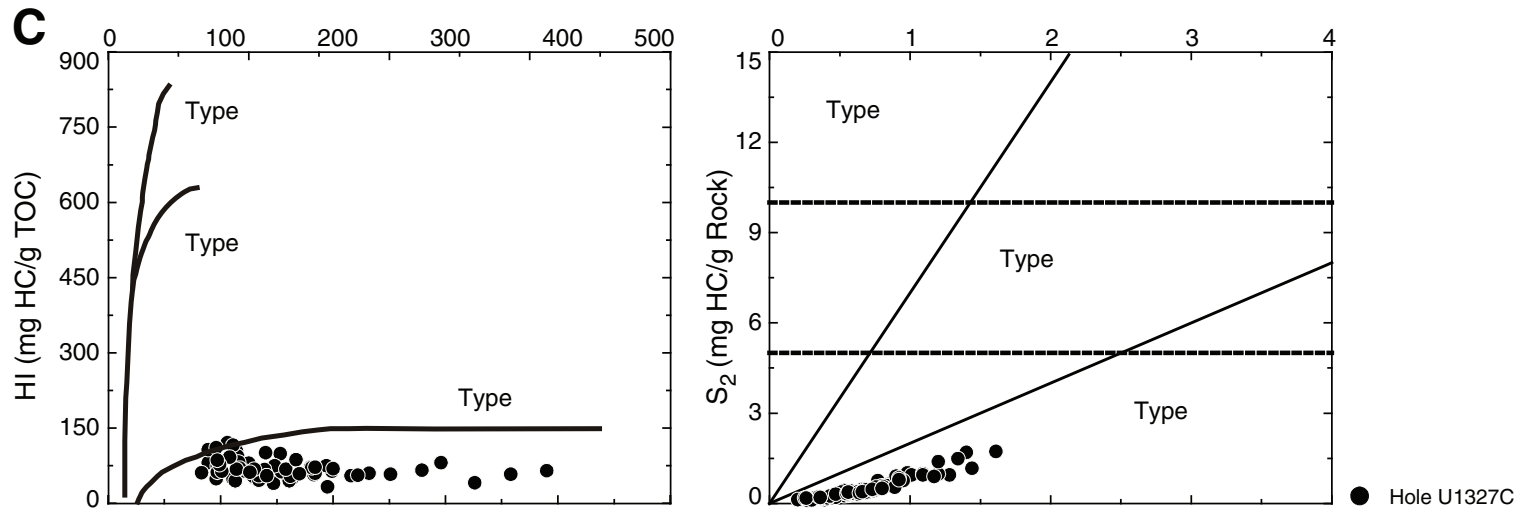
Figure F4 (continued). D. Site U1328. E. Site U1329.
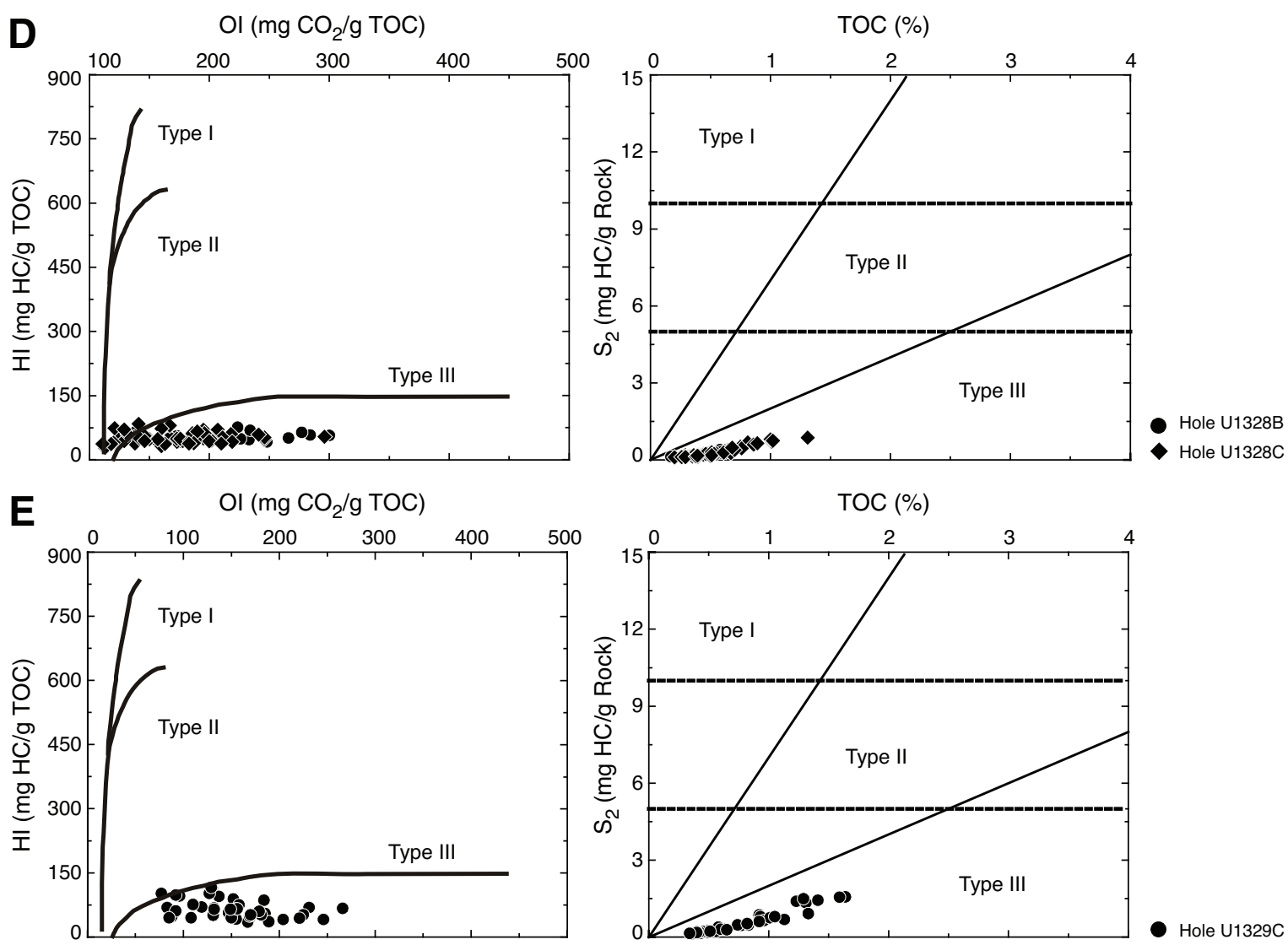
Table T1. Compositions of bulk sediments. (See table note.) (Continued on next four pages.)

\begin{tabular}{|c|c|c|c|c|c|c|c|c|c|c|c|c|c|c|}
\hline \multirow{3}{*}{$\begin{array}{l}\text { Core, section, } \\
\text { interval }(\mathrm{cm})\end{array}$} & \multirow{3}{*}{$\begin{array}{l}\text { Depth } \\
\text { (mbsf) }\end{array}$} & \multirow{2}{*}{\multicolumn{3}{|c|}{ Major elements (wt\%) }} & \multicolumn{8}{|c|}{ Rock-Eval pyrolysis } & & \\
\hline & & & & & \multirow{2}{*}{$\begin{array}{c}\mathrm{S}_{2} \\
\text { (mg HC/ } \\
\text { g rock) }\end{array}$} & \multirow{2}{*}{$\begin{array}{c}\mathrm{S}_{3} \\
\text { (mg rock) }\end{array}$} & \multirow{2}{*}{$\begin{array}{l}T_{\max } \\
\left({ }^{\circ} \mathrm{C}\right)\end{array}$} & & $\underset{\substack{\mathrm{HI} \\
\mathrm{HC}}}{2}$ & $\mathrm{Ol}$ & & & Isotope & (\%o) \\
\hline & & TC & $\mathrm{TN}$ & TS & & & & (wt\%) & & & TN & TS & $\delta^{13} \mathrm{C}$ & $\delta^{15} \mathrm{~N}$ \\
\hline 311-U1325B- & & & & & & & & & & & & & & \\
\hline $1 \mathrm{H}-1,65-80$ & 0.7 & 2.12 & 0.22 & 0.46 & 2.61 & 2.71 & 412 & 1.73 & 151 & 157 & 7.8 & 3.8 & -23.2 & 6.9 \\
\hline $1 \mathrm{H}-1,135-150$ & 1.4 & 2.05 & 0.19 & 0.48 & 1.95 & 2.82 & 415 & 1.54 & 127 & 183 & 8.1 & 3.2 & -24.1 & 6.0 \\
\hline $1 \mathrm{H}-2,65-80$ & 2.2 & 1.93 & 0.18 & 0.65 & 2.08 & 2.20 & 416 & 1.51 & 138 & 146 & 8.5 & 2.3 & -24.2 & 6.4 \\
\hline $1 \mathrm{H}-2,135-150$ & 2.9 & 1.06 & 0.06 & 0.57 & 0.28 & 0.97 & 411 & 0.42 & 67 & 231 & 7.2 & 0.7 & -24.4 & 6.1 \\
\hline $1 \mathrm{H}-3,60-75$ & 3.7 & 0.51 & 0.03 & 0.27 & 0.13 & 0.52 & 407 & 0.27 & 48 & 193 & 9.3 & 1.0 & -26.0 & 4.3 \\
\hline $1 \mathrm{H}-3,135-150$ & 4.4 & 0.86 & 0.06 & 0.28 & 0.30 & 0.85 & 410 & 0.40 & 75 & 212 & 7.1 & 1.4 & -24.8 & 4.4 \\
\hline $1 \mathrm{H}-4,65-80$ & 5.2 & 1.20 & 0.09 & 0.22 & 0.61 & 1.40 & 416 & 0.79 & 77 & 177 & 9.1 & 3.7 & -24.9 & 2.9 \\
\hline $1 \mathrm{H}-4,135-150$ & 5.9 & 0.94 & 0.07 & 0.27 & 0.38 & 0.94 & 417 & 0.55 & 69 & 171 & 8.2 & 2.0 & -24.9 & 3.1 \\
\hline $1 \mathrm{H}-5,65-80$ & 6.7 & 1.13 & 0.08 & 0.09 & 0.50 & 1.05 & 422 & 0.61 & 82 & 172 & 7.8 & 6.5 & -24.2 & 7.5 \\
\hline $1 \mathrm{H}-5,135-150$ & 7.4 & 1.30 & 0.10 & 0.17 & 0.75 & 1.51 & 417 & 0.83 & 90 & 182 & 8.6 & 4.9 & -24.1 & 6.0 \\
\hline $1 \mathrm{H}-6,65-80$ & 8.2 & 0.40 & 0.02 & 0.15 & 0.10 & 0.30 & 408 & 0.12 & 83 & 250 & 5.7 & 0.8 & -24.1 & -0.5 \\
\hline $1 \mathrm{H}-6,135-150$ & 8.9 & 1.02 & 0.07 & 0.24 & 0.41 & 1.03 & 419 & 0.54 & 76 & 191 & 8.2 & 2.3 & -25.0 & 3.7 \\
\hline $2 \mathrm{H}-2,135-150$ & 12.2 & 0.25 & 0.01 & 0.04 & 0.08 & 0.15 & 344 & 0.07 & 114 & 214 & 5.0 & 1.8 & -26.0 & 5.0 \\
\hline $2 \mathrm{H}-6,135-150$ & 18.2 & 0.89 & 0.07 & 0.29 & 0.36 & 0.80 & 423 & 0.55 & 65 & 145 & 8.5 & 1.9 & -25.6 & 2.7 \\
\hline $4 \mathrm{H}-2,135-150$ & 26.7 & 1.59 & 0.10 & 0.41 & 0.68 & 1.49 & 404 & 0.84 & 81 & 177 & 8.2 & 2.1 & -24.2 & 3.7 \\
\hline $4 \mathrm{H}-5,135-150$ & 31.2 & 2.05 & 0.15 & 0.38 & 1.53 & 2.15 & 412 & 1.26 & 121 & 171 & 8.7 & 3.3 & -23.3 & 5.0 \\
\hline $5 \mathrm{H}-2,127-142$ & 36.3 & 1.07 & 0.06 & 0.73 & 0.31 & 1.20 & 401 & 0.51 & 61 & 235 & 8.8 & 0.7 & -25.9 & 0.2 \\
\hline $5 \mathrm{H}-5,135-150$ & 40.8 & 1.61 & 0.15 & 0.95 & 1.44 & 1.65 & 406 & 1.30 & 111 & 127 & 8.8 & 1.4 & -24.1 & 2.6 \\
\hline $6 \mathrm{H}-2,135-150$ & 45.9 & 1.15 & 0.08 & 0.51 & 0.30 & 1.13 & 406 & 0.56 & 54 & 202 & 7.4 & 1.1 & -25.4 & 2.9 \\
\hline $6 \mathrm{H}-5,135-150$ & 50.4 & 1.09 & 0.08 & 0.52 & 0.31 & 0.88 & 367 & 0.61 & 51 & 144 & 7.6 & 1.2 & -24.9 & 1.2 \\
\hline 7H- $2,135-150$ & 55.4 & 0.65 & 0.05 & 0.03 & 0.24 & 0.57 & 428 & 0.47 & 51 & 121 & 9.0 & 18.0 & -26.5 & 0.5 \\
\hline 7H-5, 135-150 & 59.9 & 0.76 & 0.04 & 0.25 & 0.20 & 0.86 & 428 & 0.39 & 51 & 221 & 10.5 & 1.6 & -26.0 & 2.1 \\
\hline $8 \mathrm{H}-3,125-150$ & 66.1 & 0.59 & 0.04 & 0.04 & 0.20 & 0.76 & 430 & 0.39 & 51 & 195 & 10.8 & 9.6 & -26.6 & 0.6 \\
\hline $8 \mathrm{H}-5,123-150$ & 69.1 & 0.51 & 0.04 & 0.02 & 0.14 & 0.47 & 422 & 0.34 & 41 & 138 & 8.9 & 17.3 & -26.0 & -0.7 \\
\hline $9 X-2,0-25$ & 73.0 & 0.55 & 0.05 & 0.03 & 0.19 & 0.66 & 427 & 0.47 & 40 & 140 & 9.4 & 13.5 & -26.5 & 1.6 \\
\hline $10 X-3,125-150$ & 77.2 & 1.35 & 0.11 & 0.27 & 0.59 & 1.55 & 408 & 0.87 & 68 & 178 & 7.8 & 3.2 & -24.9 & 3.7 \\
\hline $10 X-5,107-117$ & 79.9 & 0.67 & 0.06 & 0.05 & 0.26 & 0.65 & 421 & 0.58 & 45 & 112 & 9.2 & 10.7 & -26.1 & 0.5 \\
\hline $10 X-7,31-41$ & 82.0 & 0.69 & 0.05 & 0.14 & 0.22 & 0.67 & 418 & 0.37 & 59 & 181 & 8.0 & 2.7 & -25.4 & 2.7 \\
\hline $12 X-2,125-150$ & 87.3 & 0.68 & 0.05 & 0.18 & 0.22 & 0.56 & 427 & 0.46 & 48 & 122 & 9.0 & 2.6 & -26.0 & 3.0 \\
\hline $12 X-4,68-93$ & 89.7 & 0.58 & 0.04 & 0.10 & 0.14 & 0.60 & 425 & 0.30 & 47 & 200 & 7.0 & 3.0 & -26.0 & 3.0 \\
\hline $14 \mathrm{X}-2,125-150$ & 105.2 & 1.85 & 0.11 & 0.47 & 0.62 & 1.75 & 409 & 0.73 & 85 & 240 & 7.0 & 1.6 & -24.7 & 3.2 \\
\hline $14 X-5,125-131$ & 109.6 & 0.76 & 0.07 & 0.13 & 0.35 & 0.62 & 428 & 0.61 & 57 & 102 & 9.1 & 4.8 & -26.4 & -0.1 \\
\hline $14 X-6,0-25$ & 109.8 & 0.77 & 0.07 & 0.04 & 0.34 & 0.76 & 422 & 0.56 & 61 & 136 & 7.7 & 12.5 & -25.9 & 0.9 \\
\hline $15 X-2,120-150$ & 114.6 & 0.5 & 0.05 & 0.1 & 0.21 & 6 & 428 & 0.04 & 525 & 1150 & 0.9 & 0.2 & -26.6 & -1.4 \\
\hline $15 X-3,0-14$ & 114.8 & 0.50 & 0.04 & 0.19 & 0.15 & 0.38 & 427 & 0.27 & 56 & 141 & 6.4 & 1.4 & -26.5 & 2.6 \\
\hline $16 \mathrm{X}-2,120-150$ & 124.2 & 0.96 & 0.09 & 0.49 & 0.44 & 0.91 & 390 & 0.64 & 69 & 142 & 7.2 & 1.3 & -25.9 & 2.4 \\
\hline $16 X-4,25-35$ & 126.1 & 0.94 & 0.06 & 0.55 & 0.26 & 0.78 & 406 & 0.46 & 57 & 170 & 7.3 & 0.8 & -25.6 & 2.0 \\
\hline $16 \mathrm{X}-6,120-150$ & 128.0 & 1.42 & 0.07 & 0.68 & 0.27 & 1.13 & 391 & 0.51 & 53 & 222 & 7.3 & 0.8 & -26.2 & 0.2 \\
\hline $18 \mathrm{X}-1,140-150$ & 132.4 & 0.85 & 0.05 & 0.73 & 0.20 & 0.77 & 398 & 0.31 & 65 & 248 & 6.7 & 0.4 & -24.7 & 2.8 \\
\hline $18 \mathrm{X}-2,126-156$ & 133.8 & 0.83 & 0.08 & 0.58 & 0.32 & 0.81 & 376 & 0.58 & 55 & 140 & 7.1 & 1.0 & -25.2 & 1.4 \\
\hline $19 X-3,21-30$ & 143.8 & 1.35 & 0.05 & 0.66 & 0.16 & 0.91 & 415 & 0.40 & 40 & 228 & 7.5 & 0.6 & -26.9 & 1.1 \\
\hline $19 X-5,31-53$ & 145.7 & 1.16 & 0.07 & 0.21 & 0.28 & 1.10 & 423 & 0.55 & 51 & 200 & 8.0 & 2.6 & -25.5 & -1.1 \\
\hline $20 X-3,45-64$ & 153.7 & 0.87 & 0.06 & 0.06 & 0.20 & 0.89 & 433 & 0.47 & 43 & 189 & 8.0 & 8.5 & -26.4 & -1.0 \\
\hline $20 X-5,120-150$ & 156.7 & 1.06 & 0.06 & 0.3 & 0.33 & 1.20 & 418 & 0.55 & 60 & 21 & 9.0 & 1.6 & -25.8 & 1.6 \\
\hline $21 X-1,37-51$ & 160.2 & 0.77 & 0.04 & 0.14 & 0.13 & 0.70 & 425 & 0.26 & 50 & 269 & 6.8 & 1.9 & -26.0 & 2.8 \\
\hline $21 X-2,120-150$ & 161.7 & 0.54 & 0.04 & 0.06 & 0.16 & 0.71 & 431 & 0.33 & 48 & 215 & 7.5 & 5.5 & -25.9 & 1.1 \\
\hline $23 X-1,120-150$ & 171.8 & 0.76 & 0.05 & 0.08 & 0.19 & 0.93 & 420 & 0.34 & 56 & 274 & 6.5 & 4.1 & -25.9 & -1.4 \\
\hline $24 \mathrm{X}-2,0-11$ & 180.4 & 0.93 & 0.07 & 0.27 & 0.36 & 1.13 & 409 & 0.53 & 68 & 213 & 7.4 & 2.0 & -25.0 & -1.7 \\
\hline $24 X-3,120-150$ & 183.1 & 0.90 & 0.07 & 0.29 & 0.35 & 1.22 & 412 & 0.57 & 61 & 214 & 7.7 & 2.0 & -25.9 & 2.0 \\
\hline $24 X-4,0-14$ & 183.3 & 1.59 & 0.10 & 0.03 & 0.41 & 1.74 & 406 & 0.59 & 69 & 295 & 6.1 & 17.2 & -25.2 & 0.6 \\
\hline $24 X-5,112-145$ & 185.6 & 1.06 & 0.09 & 0.15 & 0.37 & 1.26 & 423 & 0.65 & 57 & 194 & 7.5 & 4.3 & -25.8 & 0.8 \\
\hline 311-U1325C- & & & & & & & & & & & & & & \\
\hline $1 \mathrm{X}-1,125-150$ & 190.2 & 0.70 & 0.05 & 0.30 & 0.29 & 0.72 & 418 & 0.43 & 67 & 167 & 9.1 & 1.4 & -25.3 & 3.1 \\
\hline $1 \mathrm{X}-2,141-150 \mathrm{~B}$ & 191.8 & 0.58 & 0.04 & 0.18 & 0.16 & 0.61 & 425 & 0.31 & 52 & 197 & 7.6 & 1.7 & -26.3 & -0.6 \\
\hline $2 X-1,0-43$ & 198.6 & 0.76 & 0.07 & 0.09 & 0.22 & 1.24 & 424 & 0.43 & 51 & 288 & 6.5 & 4.7 & -26.0 & 0.1 \\
\hline $4 X-3,0-79$ & 210.5 & 0.58 & 0.05 & 0.07 & 0.19 & 0.94 & 431 & 0.42 & 45 & 224 & 8.6 & 6.4 & -26.0 & 1.8 \\
\hline $6 X-3,88-112$ & 221.4 & 0.52 & 0.04 & 0.08 & 0.16 & 0.68 & 422 & 0.34 & 47 & 200 & 8.3 & 4.2 & -24.7 & -2.9 \\
\hline $7 X-2,0-50$ & 229.0 & 0.53 & 0.05 & 0.07 & 0.17 & 0.80 & 425 & 0.32 & 53 & 250 & 6.7 & 4.8 & -26.4 & 0.1 \\
\hline $7 X-4,69-79$ & 231.9 & 0.65 & 0.07 & 0.10 & 0.16 & 0.72 & 427 & 0.38 & 42 & 189 & 5.6 & 3.9 & -26.9 & -2.2 \\
\hline $8 X-2,62-85$ & 239.0 & 0.32 & 0.03 & 0.16 & 0.07 & 0.38 & 436 & 0.15 & 47 & 253 & 4.8 & 0.9 & -27.7 & -5.9 \\
\hline $8 X-4,0-50$ & 240.9 & 0.74 & 0.06 & 0.08 & 0.17 & 0.93 & 425 & 0.38 & 45 & 245 & 6.9 & 5.1 & -26.4 & 0.3 \\
\hline $9 \times-2,100-150$ & 249.3 & 0.69 & 0.05 & 0.10 & 0.21 & 0.71 & 426 & 0.40 & 52 & 178 & 7.5 & 4.0 & -26.9 & -0.9 \\
\hline $9 X-4,111-128$ & 252.2 & 0.33 & 0.03 & 0.17 & 0.08 & 0.31 & 420 & 0.13 & 62 & 238 & 4.8 & 0.8 & -27.0 & 0.0 \\
\hline $10 \mathrm{P}-1,6-16$ & 256.2 & 0.57 & 0.05 & 0.07 & 0.17 & 0.75 & 438 & 0.38 & 45 & 197 & 7.2 & 5.5 & -27.6 & -2.2 \\
\hline $10 \mathrm{P}-1,60-70$ & 256.8 & 0.48 & 0.04 & 0.03 & 0.17 & 0.54 & 426 & 0.32 & 53 & 169 & 7.3 & 9.3 & -27.3 & 2.5 \\
\hline
\end{tabular}


Table T1 (continued). (Continued on next page.)

\begin{tabular}{|c|c|c|c|c|c|c|c|c|c|c|c|c|c|c|}
\hline \multirow{3}{*}{$\begin{array}{l}\text { Core, section, } \\
\text { interval }(\mathrm{cm})\end{array}$} & \multirow{3}{*}{$\begin{array}{l}\text { Depth } \\
\text { (mbsf) }\end{array}$} & \multirow{2}{*}{\multicolumn{3}{|c|}{ Major elements (wt\%) }} & \multicolumn{8}{|c|}{ Rock-Eval pyrolysis } & & \\
\hline & & & & & \multirow{2}{*}{$\begin{array}{c}\mathrm{S}_{2} \\
\text { (mg HCl } \\
\text { g rock) }\end{array}$} & \multirow{2}{*}{$\begin{array}{c}\mathrm{S}_{3} \\
\text { (mg Co } \mathrm{CO}_{2} / \\
\mathrm{g} \text { rock) }\end{array}$} & \multirow{2}{*}{$\begin{array}{l}T_{\max } \\
\left({ }^{\circ} \mathrm{C}\right)\end{array}$} & \multirow{2}{*}{$\begin{array}{l}\text { TOC } \\
\text { (wt\%) }\end{array}$} & & & & & Isotope & s (\%o) \\
\hline & & TC & TN & TS & & & & & & & TN & TS & $\delta^{13} \mathrm{C}$ & $\delta^{15} \mathrm{~N}$ \\
\hline $11 X-2,105-150$ & 259.9 & 0.73 & 0.05 & 0.09 & 0.20 & 0.79 & 436 & 0.41 & 49 & 193 & 8.0 & 4.5 & -26.6 & 4.1 \\
\hline $11 X-5,0-45$ & 263.3 & 0.55 & 0.05 & 0.04 & 0.17 & 0.60 & 419 & 0.31 & 55 & 194 & 6.5 & 7.7 & -27.1 & -0.1 \\
\hline $12 X-3,0-45$ & 268.9 & 0.59 & 0.05 & 0.06 & 0.19 & 0.58 & 441 & 0.37 & 51 & 157 & 7.7 & 5.9 & -26.1 & 3.7 \\
\hline $14 X-2,0-80$ & 286.3 & 0.53 & 0.04 & 0.04 & 0.17 & 0.57 & 419 & 0.31 & 55 & 184 & 7.2 & 7.7 & -27.0 & -0.1 \\
\hline $15 X-3,0-134$ & 297.8 & 0.64 & 0.05 & 0.04 & 0.19 & 0.70 & 423 & 0.28 & 68 & 250 & 5.7 & 7.5 & -27.5 & 0.6 \\
\hline 311-U1326C- & & & & & & & & & & & & & & \\
\hline $1 \mathrm{H}-1,65-80$ & 0.7 & 0.69 & 0.04 & 0.70 & 0.21 & 0.50 & 424 & 0.49 & 43 & 102 & 12.3 & 0.7 & -27.0 & 0.2 \\
\hline $1 \mathrm{H}-1,135-150$ & 1.4 & 0.80 & 0.04 & 0.39 & 0.11 & 0.51 & 405 & 0.27 & 41 & 189 & 6.8 & 0.7 & -26.8 & 2.5 \\
\hline $1 \mathrm{H}-2,65-80$ & 2.2 & 1.75 & 0.06 & 1.18 & 0.36 & 0.99 & 342 & 0.53 & 68 & 187 & 9.1 & 0.4 & -25.8 & 2.4 \\
\hline $1 \mathrm{H}-2,135-150$ & 2.9 & 1.25 & 0.08 & 1.07 & 0.29 & 1.00 & 391 & 0.56 & 52 & 179 & 7.1 & 0.5 & -25.7 & 1.1 \\
\hline $1 \mathrm{H}-3,60-72$ & 3.7 & 1.42 & 0.05 & 1.15 & 0.15 & 0.80 & 408 & 0.41 & 37 & 195 & 7.9 & 0.4 & -25.4 & 2.0 \\
\hline $2 \mathrm{H}-1,60-75$ & 4.6 & 1.21 & 0.06 & 0.72 & 0.22 & 0.75 & 394 & 0.44 & 50 & 170 & 7.5 & 0.6 & -25.2 & 1.4 \\
\hline $2 \mathrm{H}-1,135-150$ & 5.3 & 0.85 & 0.06 & 0.82 & 0.26 & 0.66 & 419 & 0.47 & 55 & 140 & 7.7 & 0.6 & -26.5 & 1.5 \\
\hline $2 \mathrm{H}-2,65-80$ & 6.1 & 1.36 & 0.10 & 0.34 & 0.51 & 1.48 & 381 & 0.75 & 68 & 197 & 7.8 & 2.2 & -24.0 & 3.4 \\
\hline $2 \mathrm{H}-2,135-150$ & 6.8 & 0.90 & 0.10 & 0.59 & 0.48 & 1.06 & 340 & 0.68 & 71 & 156 & 6.6 & 1.1 & -24.5 & 2.7 \\
\hline $2 \mathrm{H}-3,60-75$ & 7.6 & 0.89 & 0.05 & 0.79 & 0.16 & 0.99 & 402 & 0.35 & 46 & 283 & 6.7 & 0.4 & -25.5 & 2.5 \\
\hline $2 \mathrm{H}-3,135-150$ & 8.3 & 1.37 & 0.08 & 0.41 & 0.26 & 0.98 & 415 & 0.52 & 50 & 188 & 6.8 & 1.3 & -25.7 & 1.4 \\
\hline $2 \mathrm{H}-4,65-80$ & 9.1 & 0.84 & 0.06 & 0.34 & 0.25 & 0.75 & 422 & 0.43 & 58 & 174 & 7.3 & 1.3 & -26.0 & 0.6 \\
\hline $2 \mathrm{H}-5,135-150$ & 11.3 & 1.49 & 0.08 & 0.13 & 0.30 & 1.09 & 409 & 0.46 & 65 & 237 & 5.8 & 3.7 & -25.0 & 1.5 \\
\hline $2 \mathrm{H}-6,135-150$ & 12.8 & 0.83 & 0.06 & 0.26 & 0.28 & 0.85 & 422 & 0.45 & 62 & 189 & 7.1 & 1.7 & -25.1 & 1.8 \\
\hline $3 \mathrm{H}-2,135-150$ & 16.3 & 1.41 & 0.08 & 0.26 & 0.34 & 1.13 & 408 & 0.55 & 62 & 205 & 6.5 & 2.1 & -24.8 & 2.7 \\
\hline $3 \mathrm{H}-4,135-150$ & 19.3 & 0.79 & 0.05 & 0.19 & 0.21 & 0.97 & 410 & 0.39 & 54 & 249 & 7.4 & 2.0 & -24.7 & 3.1 \\
\hline $4 \mathrm{H}-2,135-150$ & 25.8 & 0.73 & 0.05 & 0.29 & 0.20 & 0.73 & 423 & 0.47 & 43 & 155 & 8.7 & 1.6 & -26.9 & 0.1 \\
\hline $6 \mathrm{X}-2,125-150$ & 42.9 & 0.64 & 0.05 & 0.20 & 0.23 & 0.45 & 432 & 0.49 & 47 & 92 & 10.7 & 2.5 & -26.5 & 1.7 \\
\hline $6 X-4,83-96$ & 44.9 & 0.93 & 0.05 & 0.45 & 0.27 & 0.54 & 426 & 0.53 & 51 & 102 & 9.8 & 1.2 & -26.2 & -0.1 \\
\hline $7 X-1,93-133$ & 50.8 & 0.79 & 0.07 & 0.21 & 0.26 & 0.50 & 427 & 0.51 & 51 & 98 & 7.7 & 2.4 & -26.2 & 0.7 \\
\hline 7X-3, 74-101 & 53.4 & 0.78 & 0.07 & 0.15 & 0.27 & 0.41 & 430 & 0.53 & 51 & 77 & 7.5 & 3.5 & -27.2 & -1.2 \\
\hline $9 X-2,0-25$ & 70.7 & 0.83 & 0.06 & 0.26 & 0.28 & 0.60 & 428 & 0.62 & 45 & 97 & 10.0 & 2.4 & -27.2 & 1.0 \\
\hline $9 X-3,66-91$ & 72.9 & 0.79 & 0.07 & 0.18 & 0.26 & 0.52 & 427 & 0.55 & 47 & 95 & 8.2 & 3.1 & -27.4 & -0.4 \\
\hline $10 \mathrm{H}-2,0-30$ & 80.4 & 0.52 & 0.05 & 0.14 & 0.18 & 0.43 & 425 & 0.39 & 46 & 110 & 8.1 & 2.7 & -26.5 & 1.4 \\
\hline 311-U1326D- & & & & & & & & & & & & & & \\
\hline $2 X-2,125-150$ & 91.3 & 0.56 & 0.05 & 0.08 & 0.42 & 0.69 & 358 & 0.53 & 79 & 130 & 9.8 & 6.9 & -26.9 & -0.4 \\
\hline $2 X-5,104-144$ & 95.6 & 0.89 & 0.09 & 0.22 & 0.29 & 0.61 & 427 & 0.73 & 40 & 84 & 8.4 & 3.3 & -26.6 & 1.3 \\
\hline $3 X-4,61-90$ & 103.4 & 0.75 & 0.06 & 0.37 & 0.07 & 0.56 & 421 & 0.26 & 27 & 215 & 4.7 & 0.7 & -26.5 & 1.6 \\
\hline $3 X-4,90-119$ & 103.6 & 0.99 & 0.05 & 0.55 & 0.23 & 0.64 & 429 & 0.38 & 61 & 168 & 8.4 & 0.7 & -26.0 & 0.8 \\
\hline $4 X-3,40-106$ & 111.4 & 0.81 & 0.08 & 0.18 & 0.28 & 0.75 & 429 & 0.55 & 51 & 136 & 7.3 & 3.0 & -26.3 & 0.6 \\
\hline $4 X-5,126-146$ & 114.5 & 0.15 & 0.01 & 0.01 & 0.08 & 0.05 & 514 & 0.09 & 89 & 56 & 6.4 & 6.3 & -24.5 & 1.1 \\
\hline $5 X-3,102-150$ & 121.3 & 0.52 & 0.05 & 0.06 & 0.15 & 0.44 & 431 & 0.41 & 37 & 107 & 8.9 & 6.9 & -26.6 & -2.5 \\
\hline $5 X-4,115-150$ & 122.9 & 0.36 & 0.03 & 0.06 & 2 & 0.28 & 430 & 0.22 & 55 & 127 & 7.9 & 3.8 & -26.4 & 1.9 \\
\hline $6 X-3,100-113$ & 131.1 & 0.16 & 0.01 & 0.04 & 0 & 0.12 & 516 & 0.0 & 62 & 150 & 6.7 & 1.9 & -25.2 & 2.6 \\
\hline $6 \mathrm{X}-4,90-132$ & 132.2 & 0.42 & 0.03 & 0.04 & 0.09 & 0.38 & 431 & 0.19 & 47 & 200 & 6.3 & 4.3 & -26.2 & -1.4 \\
\hline 7X-1, 73-96 & 137.4 & 0.14 & 0.01 & 0.01 & 0.09 & 0.17 & 548 & 0.09 & 100 & 189 & 6.9 & 7.1 & -26.2 & -2.5 \\
\hline $7 X-3,0-50$ & 139.0 & 0.64 & 0.05 & 0.06 & 0.16 & 0.76 & 436 & 0.40 & 40 & 190 & 7.8 & 6.5 & -26.8 & -0.7 \\
\hline $8 X-2,36-86$ & 148.4 & 1.31 & 0.07 & 0.21 & 0.20 & 1.13 & 411 & 0.46 & 43 & 246 & 7.1 & 2.2 & -25.6 & 0.2 \\
\hline $8 X-2,86-96$ & 148.7 & 0.88 & 0.04 & 1.1 & 0 & 0.69 & 326 & 0.22 & 45 & 314 & 5.5 & 0.2 & -25.1 & 4.3 \\
\hline $9 X-1,84-134$ & 157.0 & 1.43 & 0.12 & 0.50 & 0.66 & 1.89 & 414 & 0.97 & 68 & 195 & 8.1 & 2.0 & -25.6 & 3.5 \\
\hline $9 X-4,35-48$ & 158.5 & 0.71 & 0.06 & 0.36 & 0.22 & 0.99 & 399 & 0.37 & 59 & 268 & 6.1 & 1.0 & -25.5 & 1.2 \\
\hline $10 \times-2,97-142$ & 168.3 & 1.16 & 0.09 & 0.28 & 0.38 & 1.72 & 419 & 0.75 & 51 & 229 & 8.2 & 2.6 & -25.0 & 1.8 \\
\hline $10 X-6,0-66$ & 172.4 & 0.69 & 0.06 & 0.19 & 0.20 & 0.74 & 426 & 0.43 & 47 & 172 & 6.7 & 2.3 & -26.1 & 1.3 \\
\hline $11 X-1,38-49$ & 175.6 & 0.51 & 0.05 & 0.20 & 0.15 & 0.42 & 347 & 0.29 & 52 & 145 & 5.8 & 1.5 & -26.5 & -0.9 \\
\hline $11 X-4,110-150$ & 179.9 & 0.83 & 0.07 & 0.22 & 0.29 & 1.17 & 429 & 0.46 & 63 & 254 & 7.0 & 2.1 & -25.4 & -2.8 \\
\hline $12 X-1,75-125$ & 185.8 & 1.16 & 0.08 & 0.15 & 0.33 & 1.22 & 420 & 0.57 & 58 & 214 & 6.9 & 3.8 & -25.8 & 2.3 \\
\hline $12 X-3,100-150^{*}$ & 188.8 & 1.43 & 0.08 & 0.04 & 0.26 & 1.28 & 414 & 0.41 & 63 & 312 & 5.1 & 9.5 & -24.9 & 1.2 \\
\hline $12 X-3,100-150$ & 188.8 & 0.29 & 0.03 & 0.11 & 0.13 & 0.31 & 317 & 0.15 & 87 & 207 & 5.6 & 1.4 & -25.8 & -1.7 \\
\hline $13 X-1,100-110$ & 195.5 & 0.80 & 0.07 & 0.12 & 0.28 & 0.88 & 344 & 0.47 & 60 & 187 & 6.5 & 3.9 & -26.0 & -0.1 \\
\hline $13 X-4,60-112$ & 198.9 & 0.80 & 0.05 & 0.10 & 0.16 & 0.88 & 420 & 0.32 & 50 & 275 & 6.0 & 3.1 & -25.5 & -1.7 \\
\hline $14 X-3,58-73$ & 206.9 & 0.34 & 0.02 & 0.06 & 0.06 & 0.24 & 332 & 0.14 & 43 & 171 & 5.8 & 2.2 & -26.3 & 1.4 \\
\hline $14 X-5,0-50$ & 208.8 & 0.88 & 0.08 & 0.09 & 0.30 & 0.94 & 423 & 0.53 & 57 & 177 & 7.0 & 5.8 & -25.6 & -0.9 \\
\hline $15 X-3,100-150$ & 217.6 & 0.41 & 0.04 & 0.07 & 0.15 & 0.43 & 426 & 0.27 & 56 & 159 & 7.5 & 4.1 & -26.3 & -0.3 \\
\hline $15 X-5,50-100$ & 220.1 & 0.56 & 0.06 & 0.05 & 0.18 & 0.56 & 428 & 0.40 & 45 & 140 & 6.9 & 7.7 & -27.2 & 2.6 \\
\hline $16 X-3,0-50$ & 226.6 & 0.61 & 0.07 & 0.06 & 0.21 & 0.72 & 425 & 0.34 & 62 & 212 & 5.2 & 5.7 & -27.2 & -1.0 \\
\hline $16 X-5,0-52$ & 229.2 & 0.47 & 0.05 & 0.05 & 0.20 & 0.43 & 428 & 0.42 & 48 & 102 & 8.6 & 8.8 & -26.5 & -0.5 \\
\hline $17 X-3,0-100$ & 236.4 & 0.51 & 0.05 & 0.05 & 0.23 & 0.47 & 351 & 0.27 & 85 & 174 & 6.0 & 5.0 & -26.5 & 2.8 \\
\hline $18 X-3,0-90$ & 245.1 & 0.98 & 0.08 & 0.12 & 0.24 & 0.86 & 336 & 0.50 & 48 & 172 & 6.3 & 4.0 & -26.0 & 0.7 \\
\hline $18 X-3,90-120$ & 245.8 & 0.33 & 0.03 & 0.07 & 0.10 & 0.31 & 305 & 0.10 & 100 & 310 & 3.3 & 1.4 & -24.9 & -3.5 \\
\hline
\end{tabular}


Table T1 (continued). (Continued on next page.)

\begin{tabular}{|c|c|c|c|c|c|c|c|c|c|c|c|c|c|c|}
\hline \multirow{3}{*}{$\begin{array}{l}\text { Core, section, } \\
\text { interval }(\mathrm{cm})\end{array}$} & \multirow{3}{*}{$\begin{array}{l}\text { Depth } \\
\text { (mbsf) }\end{array}$} & \multirow{2}{*}{\multicolumn{3}{|c|}{ Major elements (wt\%) }} & \multicolumn{8}{|c|}{ Rock-Eval pyrolysis } & & \\
\hline & & & & & \multirow{2}{*}{$\begin{array}{c}\mathrm{S}_{2} \\
\text { (mg HC/ } \\
\text { g rock) }\end{array}$} & \multirow{2}{*}{$\begin{array}{c}\mathrm{S}_{3} \\
\text { (mg CO } \\
\text { g rock) }\end{array}$} & \multirow{2}{*}{$\begin{array}{l}T_{\max } \\
\left({ }^{\circ} \mathrm{C}\right)\end{array}$} & & $\underset{(\mathrm{mI}}{\mathrm{HI}}$ & $\mathrm{Ol}^{\mathrm{O}}$ & & & Isotope & s (\%o) \\
\hline & & TC & $\mathrm{TN}$ & TS & & & & $(w t \%)$ & & & N & TS & $\delta^{13} \mathrm{C}$ & $\delta^{15} \mathrm{~N}$ \\
\hline $19 X-2,0-83$ & 254.0 & 0.89 & 0.07 & 0.20 & 0.22 & 1.01 & 421 & 0.40 & 55 & 252 & 6.1 & 2.0 & -26.3 & 3.8 \\
\hline $20 X-2,33-45$ & 263.7 & 0.68 & 0.05 & 0.17 & 0.12 & 1.50 & 323 & 0.28 & 43 & 536 & 6.2 & 1.6 & -25.5 & 0.3 \\
\hline $20 X-4,50-150$ & 266.0 & 0.78 & 0.07 & 0.16 & 0.38 & 0.98 & 336 & 0.50 & 76 & 196 & 6.8 & 3.2 & -26.1 & 1.2 \\
\hline $20 X-6,0-100$ & 268.5 & 0.63 & 0.06 & 0.07 & 0.23 & 0.86 & 428 & 0.39 & 59 & 221 & 6.2 & 5.7 & -27.3 & 2.8 \\
\hline 311-U1327C- & & & & & & & & & & & & & & \\
\hline $1 \mathrm{H}-1,140-150$ & 1.5 & 1.12 & 0.09 & 0.46 & 0.76 & 1.18 & 411 & 0.77 & 99 & 153 & 8.9 & 1.7 & -24.2 & 7.4 \\
\hline $1 \mathrm{H}-3,140-150$ & 4.5 & 0.92 & 0.03 & 0.09 & 0.17 & 0.67 & 419 & 0.31 & 55 & 216 & 11.9 & 3.3 & -25.8 & 4.5 \\
\hline $2 \mathrm{H}-1,140-150$ & 7.6 & 0.73 & 0.04 & 0.03 & 0.14 & 0.86 & 326 & 0.24 & 58 & 358 & 6.7 & 7.3 & -25.6 & 6.2 \\
\hline $2 \mathrm{H}-3,140-150$ & 10.6 & 0.71 & 0.04 & 0.06 & 0.13 & 0.78 & 422 & 0.20 & 65 & 390 & 5.6 & 3.6 & -26.5 & 2.1 \\
\hline $2 \mathrm{H}-5,140-150$ & 13.6 & 0.78 & 0.04 & 0.05 & 0.11 & 0.88 & 413 & 0.27 & 41 & 326 & 7.1 & 5.2 & -26.0 & 5.0 \\
\hline $2 \mathrm{H}-7,54-64$ & 15.7 & 1.58 & 0.08 & 0.01 & 0.38 & 1.46 & 407 & 0.63 & 60 & 232 & 7.7 & 66.9 & -24.1 & 5.5 \\
\hline $3 \mathrm{H}-2,140-150$ & 18.6 & 1.96 & 0.10 & 0.00 & 0.43 & 1.57 & 403 & 0.53 & 81 & 296 & 5.6 & 219.0 & -23.8 & 5.4 \\
\hline $3 \mathrm{H}-4,140-150$ & 21.6 & 0.56 & 0.04 & 0.05 & 0.20 & 0.64 & 333 & 0.39 & 51 & 164 & 9.8 & 8.4 & -24.9 & 4.5 \\
\hline $3 \mathrm{H}-6,140-150$ & 24.6 & 0.84 & 0.06 & 0.48 & 0.24 & 0.77 & 394 & 0.42 & 57 & 183 & 7.6 & 0.9 & -24.5 & 4.5 \\
\hline $4 \mathrm{H}-2,135-150$ & 28.0 & 0.99 & 0.04 & 0.59 & 0.13 & 0.76 & 404 & 0.39 & 33 & 195 & 10.8 & 0.7 & -25.9 & 2.0 \\
\hline $4 \mathrm{H}-5,135-150$ & 32.5 & 0.64 & 0.03 & 0.76 & 0.17 & 0.61 & 417 & 0.38 & 45 & 161 & 11.5 & 0.5 & -25.6 & 4.5 \\
\hline $5 \mathrm{H}-2,135-150$ & 37.5 & 0.76 & 0.05 & 0.12 & 0.29 & 0.74 & 416 & 0.57 & 51 & 130 & 11.2 & 4.9 & -25.9 & 4.6 \\
\hline $5 \mathrm{H}-5,135-150$ & 42.0 & 0.50 & 0.03 & 0.11 & 0.12 & 0.44 & 415 & 0.30 & 40 & 147 & 10.3 & 2.8 & -25.9 & 4.4 \\
\hline 7H- $2,135-150$ & 49.0 & 0.75 & 0.04 & 0.23 & 0.27 & 0.53 & 424 & 0.55 & 49 & 96 & 13.4 & 2.4 & -27.0 & 2.6 \\
\hline 7H-5, 135-150 & 53.5 & 0.69 & 0.05 & 0.26 & 0.26 & 0.51 & 425 & 0.53 & 49 & 96 & 11.3 & 2.1 & -25.8 & 3.6 \\
\hline $8 \mathrm{H}-2,120-150$ & 58.5 & 0.91 & 0.07 & 0.03 & 0.43 & 0.80 & 422 & 0.69 & 62 & 116 & 9.3 & 26.5 & -26.0 & 3.0 \\
\hline $8 \mathrm{H}-5,125-150$ & 63.0 & 0.70 & 0.06 & 0.08 & 0.31 & 0.70 & 422 & 0.63 & 49 & 111 & 10.5 & 8.2 & -26.1 & 4.1 \\
\hline $9 \mathrm{H}-2,120-150$ & 68.0 & 0.51 & 0.05 & 0.44 & 0.22 & 0.54 & 413 & 0.3 & 63 & 154 & 7.6 & 0.8 & -25.9 & 3.3 \\
\hline $9 \mathrm{H}-5,125-150$ & 72.5 & 1.17 & 0.10 & 1.10 & 0.64 & 1.26 & 376 & 0.85 & 75 & 148 & 8.3 & 0.8 & -24.7 & 3.2 \\
\hline $10 \mathrm{H}-2,125-150$ & 77.5 & 1.19 & 0.07 & 0.64 & 0.38 & 0.96 & 406 & 0.70 & 54 & 137 & 9.9 & 1.1 & -24.8 & 3.6 \\
\hline $10 \mathrm{H}-5,125-150$ & 80.6 & 1.14 & 0.07 & 0.72 & 0.43 & 0.94 & 410 & 0.74 & 58 & 127 & 10.3 & 1.0 & -25.1 & 3.0 \\
\hline $11 \mathrm{H}-2,125-150$ & 87.0 & 0.88 & 0.08 & 0.63 & 0.47 & 0.83 & 408 & 0.66 & 71 & 126 & 8.7 & 1.1 & -24.8 & 3.3 \\
\hline $11 \mathrm{H}-5,125-150$ & 91.5 & 1.34 & 0.09 & 0.44 & 0.47 & 1.47 & 414 & 0.74 & 64 & 199 & 8.5 & 1.7 & -24.3 & 4.6 \\
\hline $13 X-2,115-150$ & 105.3 & 1.24 & 0.13 & 0.63 & 0.93 & 1.12 & 412 & 0.99 & 94 & 113 & 7.8 & 1.6 & -24.9 & 3.3 \\
\hline $13 X-5,71-101$ & 109.0 & 0.76 & 0.07 & 0.24 & 0.30 & 0.91 & 417 & 0.56 & 54 & 162 & 8.5 & 2.3 & -24.8 & 4.2 \\
\hline $13 X-6,110-150$ & 110.8 & 0.86 & 0.08 & 0.35 & 0.37 & 0.88 & 417 & 0.68 & 54 & 129 & 8.8 & 1.9 & -24.8 & 4.5 \\
\hline $14 \mathrm{X}-2,115-150$ & 114.9 & 0.55 & 0.06 & 0.14 & 0.19 & 0.55 & 418 & 0.41 & 46 & 134 & 7.1 & 2.9 & -25.5 & 3.4 \\
\hline $14 \mathrm{X}-4,102-112$ & 117.7 & 0.82 & 0.08 & 0.36 & 0.47 & 0.81 & 408 & 0.68 & 69 & 119 & 8.5 & 1.9 & -24.2 & 4.0 \\
\hline $14 \mathrm{X}-4,112-122$ & 117.8 & 0.72 & 0.07 & 0.27 & 0.35 & 0.70 & 408 & 0.53 & 66 & 132 & 7.5 & 2.0 & -25.0 & -1.3 \\
\hline $15 \mathrm{P}-1,0-25$ & 121.8 & 0.84 & 0.06 & 0.59 & 0.34 & 0.81 & 408 & 0.58 & 59 & 140 & 9.4 & 1.0 & -24.9 & 4.1 \\
\hline $15 \mathrm{P}-1,25-50$ & 122.1 & 0.71 & 0.05 & 0.53 & 0.24 & 0.59 & 409 & 0.44 & 55 & 134 & 8.1 & 0.8 & -24.9 & 4.2 \\
\hline $16 X-2,120-150$ & 126.7 & 1.01 & 0.08 & 0.32 & 9 & 0.94 & 417 & 0 & 74 & 118 & 9.5 & 2.5 & -24.7 & 3.2 \\
\hline $16 \mathrm{X}-4,120-150$ & 129.7 & 0.62 & 0.07 & 0.04 & 0.21 & 0.53 & 422 & 0.47 & 45 & 113 & 7.2 & 11.6 & -25.7 & 3.3 \\
\hline $17 X-2,0-10$ & 132.4 & 1.37 & 0.11 & 0.48 & 1.02 & 1.12 & 419 & 0.98 & 104 & 114 & 8.9 & 2.0 & -23.9 & 5.1 \\
\hline $17 X-2,10-20$ & 132.5 & 1.36 & 0.12 & 0.59 & 0.95 & 1.16 & 419 & 1.01 & 94 & 115 & 8.6 & 1.7 & -23.7 & 5.5 \\
\hline $17 X-3,64-70$ & 133.9 & 1.17 & 0.10 & 0.28 & 0.76 & 1.19 & 413 & 0.95 & 80 & 125 & 9.9 & 3.4 & -24.1 & 5.0 \\
\hline $17 X-4,65-74$ & 134.6 & 1.09 & 0.08 & 0.17 & 052 & 0.99 & 422 & 0.81 & 64 & 122 & 9.9 & 4.8 & -25.1 & 4.2 \\
\hline $18 X-2,0-10$ & 142.4 & 1.45 & 0.14 & 0.98 & 11 & 1.29 & 411 & 1.44 & 81 & 89 & 10.5 & 1.5 & -24.7 & 1.6 \\
\hline $18 X-2,0-10$ & 142.4 & 1.40 & 0.13 & 1.07 & 0.95 & 1.24 & 412 & 1.28 & 74 & 97 & 9.6 & 1.2 & -24.4 & 4.0 \\
\hline $18 \mathrm{X}-2,120-150$ & 143.7 & 1.43 & 0.14 & 1.25 & 0.98 & 1.39 & 411 & 1.20 & 82 & 116 & 8.6 & 1.0 & -24.5 & 4.3 \\
\hline $19 X-2,69-79$ & 153.1 & 1.96 & 0.13 & 0.77 & 1.70 & 1.49 & 416 & 1.40 & 121 & 106 & 11.1 & 1.8 & -23.9 & 5.3 \\
\hline $19 X-4,120-150$ & 156.0 & 0.93 & 0.08 & 0.26 & 046 & 0.96 & 414 & 0.69 & 67 & 139 & 8.6 & 2.6 & -25.5 & 1.6 \\
\hline $20 X-2,48-76$ & 162.9 & 1.40 & 0.14 & 0.43 & 1.39 & 1.33 & 421 & 1.20 & 116 & 111 & 8.9 & 2.8 & -24.6 & 5.9 \\
\hline $20 X-4,120-150$ & 165.9 & 1.12 & 0.09 & 0.81 & 0.55 & 1.37 & 410 & 0.76 & 72 & 180 & 8.4 & 0.9 & -25.2 & 3.0 \\
\hline $21 \mathrm{X}-2,113-143$ & 173.2 & 0.86 & 0.06 & 0.12 & 0.25 & 1.08 & 433 & 0.43 & 58 & 251 & 7.7 & 3.7 & -25.7 & 3.8 \\
\hline $21 X-4,81-150$ & 175.7 & 0.98 & 0.07 & 0.13 & 0.38 & 0.99 & 422 & 0.51 & 75 & 194 & 7.2 & 3.9 & -24.9 & 4.0 \\
\hline $22 \mathrm{X}-1,0-17$ & 180.2 & 0.99 & 0.06 & 0.16 & 03 & 1.31 & 421 & 0.4 & 66 & 279 & 8.4 & 2.9 & -25.3 & -0.8 \\
\hline $22 X-1,17-26$ & 180.3 & 1.02 & 0.07 & 0.40 & 0.33 & 1.01 & 418 & 0.55 & 60 & 184 & 7.4 & 1.4 & -24.6 & 4.1 \\
\hline $22 X-1,26-37$ & 180.4 & 1.00 & 0.07 & 0.10 & 0.41 & 1.05 & 424 & 0.58 & 71 & 181 & 8.4 & 5.7 & -24.7 & 4.2 \\
\hline $22 X-2,86-118$ & 182.3 & 1.15 & 0.10 & 0.14 & 0.59 & 0.98 & 419 & 0.84 & 70 & 117 & 8.8 & 6.0 & -25.0 & 3.9 \\
\hline $23 X-1,131-161$ & 191.2 & 1.36 & 0.14 & 0.31 & 0.99 & 1.18 & 411 & 1.09 & 91 & 108 & 7.6 & 3.5 & -24.7 & 1.7 \\
\hline $23 X-3,62-89$ & 193.6 & 1.25 & 0.09 & 0.64 & 0.55 & 1.28 & 408 & 0.81 & 68 & 158 & 8.6 & 1.3 & -24.5 & 3.9 \\
\hline $25 X-1,51-61$ & 199.9 & 0.39 & 0.04 & 0.11 & 0.18 & 0.46 & 432 & 0.25 & 72 & 184 & 6.4 & 2.3 & -25.2 & 4.1 \\
\hline $25 \mathrm{X}-1,61-71$ & 200.0 & 0.34 & 0.04 & 0.16 & 0.18 & 0.52 & 425 & 0.26 & 69 & 200 & 7.2 & 1.6 & -25.8 & 4.6 \\
\hline $25 X-3,0-25$ & 201.4 & 0.61 & 0.05 & 0.26 & 0.20 & 0.80 & 426 & 0.36 & 56 & 222 & 8.0 & 1.4 & -26.1 & -1.1 \\
\hline $26 \mathrm{X}-2,84-114$ & 211.4 & 0.69 & 0.06 & 0.73 & 0.38 & 0.73 & 410 & 0.56 & 68 & 130 & 9.2 & 0.8 & -25.0 & 1.6 \\
\hline $26 \mathrm{X}-5,120-150$ & 215.9 & 1.67 & 0.13 & 0.38 & 0.95 & 1.82 & 416 & 1.09 & 87 & 167 & 8.7 & 2.9 & -24.3 & 5.1 \\
\hline $27 X-1,108-138$ & 219.7 & 1.35 & 0.09 & 0.42 & 0.91 & 1.26 & 419 & 0.90 & 101 & 140 & 9.9 & 2.1 & -24.1 & 4.1 \\
\hline $27 X-3,0-10$ & 221.3 & 0.87 & 0.07 & 0.29 & 0.39 & 0.61 & 418 & 0.63 & 62 & 97 & 9.7 & 2.2 & -24.5 & 3.8 \\
\hline $27 X-3,10-20$ & 221.4 & 0.83 & 0.07 & 0.18 & 0.38 & 0.64 & 419 & 0.56 & 68 & 114 & 8.0 & 3.1 & -23.9 & 4.0 \\
\hline $27 X-4,114-144$ & 224.0 & 28 & 11 & 30 & 5 & 0 & 0 & 12 & 92 & 108 & 3.6 & 0 & - & 2.6 \\
\hline
\end{tabular}


Table T1 (continued). (Continued on next page.)

\begin{tabular}{|c|c|c|c|c|c|c|c|c|c|c|c|c|c|c|}
\hline \multirow{3}{*}{$\begin{array}{l}\text { Core, section, } \\
\text { interval }(\mathrm{cm})\end{array}$} & \multirow{3}{*}{$\begin{array}{l}\text { Depth } \\
\text { (mbsf) }\end{array}$} & \multirow{2}{*}{\multicolumn{3}{|c|}{ Major elements (wt\%) }} & \multicolumn{8}{|c|}{ Rock-Eval pyrolysis } & & \\
\hline & & & & & \multirow{2}{*}{$\begin{array}{c}\mathrm{S}_{2} \\
\text { (mg HC/ } \\
\text { g rock) }\end{array}$} & \multirow{2}{*}{$\begin{array}{c}\mathrm{S}_{3} \\
\text { (mg CO } \\
\text { g rock) }\end{array}$} & \multirow{2}{*}{$\begin{array}{l}T_{\max } \\
\left({ }^{\circ} \mathrm{C}\right)\end{array}$} & \multirow{2}{*}{$\begin{array}{l}\text { TOC } \\
\text { (wt\%) }\end{array}$} & $\underset{(\mathrm{mgl}}{\mathrm{HC}}$ & $\begin{array}{c}\mathrm{Ol} \\
\mathrm{COCO}\end{array}$ & & & Isotope & s (\%o) \\
\hline & & TC & $\mathrm{TN}$ & TS & & & & & & & TN & TS & $\delta^{13} \mathrm{C}$ & $\delta^{15} \mathrm{~N}$ \\
\hline $28 \mathrm{X}-1,120-150$ & 229.5 & 0.93 & 0.08 & 0.14 & 0.35 & 0.90 & 425 & 0.64 & 55 & 141 & 8.3 & 4.6 & -24.6 & 4.0 \\
\hline $28 X-3,96-125$ & 232.1 & 1.01 & 0.07 & 0.13 & 0.39 & 1.12 & 415 & 0.66 & 59 & 170 & 10.0 & 5.2 & -25.3 & 3.2 \\
\hline $29 \mathrm{X}-2,120-150$ & 240.6 & 1.04 & 0.10 & 0.33 & 0.54 & 0.74 & 409 & 0.89 & 61 & 83 & 8.6 & 2.7 & -25.0 & 0.6 \\
\hline $30 \times-2,88-118$ & 249.9 & 1.69 & 0.15 & 0.65 & 1.73 & 1.43 & 420 & 1.61 & 107 & 89 & 10.6 & 2.5 & -23.8 & 4.3 \\
\hline $31 X-4,120-150$ & 262.9 & 1.64 & 0.14 & 0.56 & 1.49 & 1.28 & 418 & 1.34 & 111 & 96 & 9.6 & 2.4 & -24.2 & 2.8 \\
\hline $32 X-4,120-150$ & 272.6 & 0.92 & 0.08 & 0.24 & 0.47 & 0.74 & 416 & 0.73 & 64 & 101 & 9.6 & 3.0 & -24.6 & 3.4 \\
\hline $33 X-4,120-150$ & 282.2 & 1.42 & 0.14 & 0.98 & 0.90 & 1.16 & 409 & 1.17 & 77 & 99 & 8.6 & 1.2 & -24.4 & 1.8 \\
\hline $34 X-4,120-150$ & 291.8 & 1.35 & 0.11 & 0.79 & 0.79 & 0.89 & 414 & 0.92 & 86 & 97 & 8.5 & 1.2 & -23.7 & 3.2 \\
\hline $35 X-2,120-150$ & 298.4 & 1.41 & 0.10 & 0.39 & 0.50 & 1.01 & 423 & 0.80 & 62 & 126 & 8.2 & 2.0 & -25.2 & 1.7 \\
\hline 311-U1328B- & & & & & & & & & & & & & & \\
\hline $1 \mathrm{H}-1,40-50$ & 0.5 & 1.16 & 0.06 & 1.22 & 0.23 & 1.14 & 403 & 0.49 & 47 & 233 & 8.2 & 0.4 & -27.2 & 0.0 \\
\hline $1 \mathrm{H}-1,140-150$ & 1.5 & 0.90 & 0.04 & 1.56 & 0.18 & 0.88 & 311 & 0.31 & 58 & 284 & 7.2 & 0.2 & -28.1 & 5.1 \\
\hline $1 \mathrm{H}-2,70-80$ & 2.3 & 0.90 & 0.05 & 1.06 & 0.22 & 0.88 & 408 & 0.43 & 51 & 205 & 8.3 & 0.4 & -26.1 & 1.6 \\
\hline $1 \mathrm{H}-2,140-150$ & 3.0 & 0.88 & 0.07 & 1.03 & 0.23 & 0.71 & 410 & 0.41 & 56 & 173 & 6.3 & 0.4 & -27.2 & 5.5 \\
\hline $1 \mathrm{H}-3,140-150$ & 4.5 & 0.78 & 0.05 & 0.89 & 0.17 & 0.79 & 416 & 0.35 & 49 & 226 & 7.8 & 0.4 & -26.9 & 1.4 \\
\hline $2 \mathrm{H}-1,80-100$ & 5.4 & 1.56 & 0.07 & 0.20 & 0.40 & 1.36 & 330 & 0.58 & 69 & 234 & 7.8 & 2.9 & -24.9 & 0.6 \\
\hline $4 \mathrm{P}-1,0-11$ & 14.6 & 1.16 & 0.05 & 0.13 & 0.17 & 0.90 & 427 & 0.30 & 57 & 300 & 6.0 & 2.4 & -26.2 & -0.5 \\
\hline $4 \mathrm{P}-1,23-31$ & 14.8 & 0.76 & 0.05 & 0.11 & 0.19 & 0.75 & 318 & 0.41 & 46 & 183 & 7.6 & 3.6 & -26.6 & -0.3 \\
\hline $4 P-1,36-46$ & 14.9 & 0.72 & 0.05 & 0.11 & 0.19 & 0.66 & 322 & 0.38 & 50 & 174 & 7.9 & 3.5 & -27.0 & 1.7 \\
\hline $4 P-1,50-57$ & 15.0 & 0.91 & 0.05 & 0.15 & 0.19 & 0.79 & 315 & 0.45 & 42 & 176 & 9.6 & 3.0 & -26.7 & -1.9 \\
\hline $4 P-1,61-67$ & 15.1 & 0.83 & 0.05 & 0.11 & 0.20 & 0.65 & 328 & 0.39 & 51 & 167 & 8.0 & 3.5 & -26.4 & 1.1 \\
\hline $5 \mathrm{H}-1,85-100$ & 17.4 & 0.74 & 0.06 & 0.26 & 0.21 & 1.09 & 327 & 0.41 & 51 & 266 & 7.5 & 1.6 & -25.7 & 3.5 \\
\hline $6 X-1,0-20$ & 18.5 & 7.34 & 0.03 & 0.01 & 0.13 & 0.38 & 345 & 0.17 & 76 & 224 & 5.9 & 15.6 & -27.8 & 1.1 \\
\hline $7 P-1,20-30$ & 26.3 & 0.84 & 0.05 & 0.09 & 0.16 & 0.64 & 310 & 0.31 & 52 & 206 & 6.5 & 3.6 & -27.3 & 0.5 \\
\hline $8 \mathrm{H}-2,120-150$ & 30.9 & 0.82 & 0.05 & 0.03 & 0.27 & 1.06 & 328 & 0.59 & 46 & 180 & 11.6 & 21.1 & -25.8 & 4.7 \\
\hline $8 \mathrm{H}-3,120-150$ & 32.4 & 0.67 & 0.04 & 0.06 & 0.13 & 0.77 & 319 & 0.31 & 42 & 248 & 7.9 & 4.9 & -26.7 & 0.1 \\
\hline $9 \mathrm{H}-2,109-134$ & 40.2 & 0.71 & 0.04 & 0.07 & 0.18 & 0.71 & 318 & 0.35 & 51 & 203 & 8.3 & 4.9 & -26.6 & 4.7 \\
\hline $9 \mathrm{H}-4,115-150$ & 43.1 & 2.44 & 0.09 & 0.22 & 0.41 & 1.77 & 389 & 0.64 & 64 & 277 & 7.5 & 2.9 & -24.1 & 6.3 \\
\hline $10 \mathrm{H}-2,125-150$ & 49.9 & 0.55 & 0.05 & 0.19 & 0.19 & 0.47 & 426 & 0.38 & 50 & 124 & 8.4 & 2.0 & -26.6 & 3.4 \\
\hline $10 \mathrm{H}-4,125-150$ & 52.9 & 0.85 & 0.07 & 0.24 & 0.32 & 0.87 & 425 & 0.59 & 54 & 147 & 8.4 & 2.5 & -27.6 & 1.9 \\
\hline 311-U1328C- & & & & & & & & & & & & & & \\
\hline $1 \mathrm{H}-3,125-150$ & 60.8 & 0.47 & 0.02 & 0.22 & 0.14 & 0.49 & 435 & 0.25 & 56 & 196 & 11.9 & 1.2 & -28.4 & 1.5 \\
\hline $1 \mathrm{H}-5,125-150$ & 63.8 & 0.55 & 0.05 & 0.20 & 0.16 & 0.65 & 432 & 0.36 & 44 & 181 & 7.8 & 1.8 & -26.2 & 2.2 \\
\hline $2 \mathrm{H}-2,125-150$ & 68.9 & 0.93 & 0.09 & 0.16 & 0.52 & 1.04 & 408 & 0.75 & 69 & 139 & 8.2 & 4.7 & -25.3 & 2.4 \\
\hline $2 \mathrm{H}-4,125-150$ & 71.9 & 1.08 & 0.10 & 0.17 & 0.68 & 1.14 & 409 & 0.81 & 84 & 141 & 7.8 & 4.8 & -24.0 & 4.4 \\
\hline $3 \mathrm{H}-2,125-150$ & 78.4 & 0.79 & 0.07 & 0.20 & 0.26 & 0.67 & 429 & 0.49 & 53 & 137 & 7.5 & 2.5 & -27.1 & -1.2 \\
\hline $3 \mathrm{H}-4,125-150$ & 81.4 & 0.62 & 0.04 & 0.08 & 0.14 & 0.77 & 422 & 0.26 & 54 & 296 & 6.0 & 3.1 & -26.9 & 5.8 \\
\hline $4 X-2,125-150$ & 87.9 & 0.45 & 0.04 & 0.07 & 2 & 0.48 & 428 & 0.27 & 44 & 178 & 6.8 & 4.1 & -27.9 & -0.5 \\
\hline $5 P-1,5-15$ & 92.1 & 0.60 & 0.06 & 0.06 & 0.22 & 0.60 & 423 & 0.45 & 49 & 133 & 7.0 & 7.4 & -25.4 & 0.5 \\
\hline $5 P-1,23-43$ & 92.3 & 0.66 & 0.06 & 0.41 & 0.20 & 0.70 & 432 & 0.45 & 44 & 156 & 8.0 & 1.1 & -28.0 & 0.3 \\
\hline $5 P-1,60-70$ & 92.7 & 0.55 & 0.06 & 0.03 & 0.20 & 0.61 & 427 & 0.45 & 44 & 136 & 7.5 & 13.2 & -27.6 & -1.0 \\
\hline $6 \mathrm{H}-7,125-150$ & 101.3 & 1.45 & 0.11 & 0.46 & 0.60 & 1.68 & 412 & 0.86 & 70 & 195 & 7.8 & 1.9 & -25.0 & 6.0 \\
\hline $7 X-3,115-140$ & 107.8 & 0.79 & 0.07 & 0.31 & 0.24 & 0.75 & 421 & 0.50 & 48 & 150 & 7.4 & 1.6 & -26.9 & 0.2 \\
\hline $8 X-2,120-150$ & 113.2 & 0.41 & 0.04 & 0.15 & 0.10 & 0.36 & 433 & 0.26 & 38 & 138 & 7.4 & 1.7 & -28.0 & 5.4 \\
\hline $8 X-3,97-113$ & 114.4 & 0.66 & 0.07 & 0.09 & 0.21 & 0.62 & 427 & 0.48 & 44 & 129 & 7.0 & 5.1 & -27.4 & 2.1 \\
\hline $9 \mathrm{X}-2,110-140$ & 122.7 & 0.47 & 0.03 & 0.17 & 0.10 & 0.39 & 426 & 0.20 & 50 & 195 & 5.9 & 1.2 & -27.8 & 4.6 \\
\hline $9 X-6,40-71$ & 127.3 & 0.66 & 0.07 & 0.14 & 0.20 & 0.83 & 427 & 0.48 & 42 & 173 & 7.1 & 3.4 & -27.5 & 1.2 \\
\hline $10 X-2,22-52$ & 131.5 & 1.25 & 0.09 & 0.12 & 0.39 & 1.42 & 422 & 0.65 & 60 & 218 & 7.1 & 5.4 & -26.0 & 4.7 \\
\hline $11 X-1,120-150$ & 140.7 & 1.56 & 0.13 & 0.50 & 0.71 & 2.09 & 412 & 1.01 & 70 & 207 & 7.7 & 2.0 & -25.0 & 2.8 \\
\hline $11 X-2,120-150$ & 142.2 & 1.41 & 0.13 & 0.30 & 0.80 & 1.67 & 420 & 1.00 & 80 & 167 & 7.5 & 3.3 & -25.4 & 5.4 \\
\hline $12 X-1,120-150$ & 150.3 & 0.86 & 0.07 & 0.30 & 0.23 & 0.93 & 420 & 0.44 & 52 & 211 & 6.0 & 1.5 & -26.0 & 1.2 \\
\hline $12 X-4,11-28$ & 153.6 & 0.89 & 0.07 & 0.26 & 0.25 & 0.88 & 416 & 0.51 & 49 & 173 & 7.8 & 1.9 & -25.8 & 5.2 \\
\hline $12 X-4,28-38$ & 153.7 & 1.14 & 0.09 & 0.12 & 0.31 & 1.11 & 414 & 0.63 & 49 & 176 & 6.8 & 5.3 & -26.2 & -0.7 \\
\hline $13 \mathrm{X}-1,0-10$ & 158.7 & 0.90 & 0.08 & 0.43 & 0.23 & 0.97 & 410 & 0.48 & 48 & 202 & 6.0 & 1.1 & -25.4 & 3.6 \\
\hline $13 X-1,10-20$ & 158.8 & 0.95 & 0.09 & 0.08 & 0.25 & 1.11 & 333 & 0.55 & 45 & 202 & 6.3 & 7.2 & -25.6 & 4.0 \\
\hline $13 X-2,120-150$ & 161.4 & 1.30 & 0.09 & 0.18 & 0.43 & 1.35 & 412 & 0.69 & 62 & 196 & 7.5 & 3.9 & -25.6 & 2.2 \\
\hline $14 X-3,120-150$ & 172.4 & 1.51 & 0.13 & 0.37 & 0.65 & 1.42 & 409 & 0.89 & 73 & 160 & 7.1 & 2.4 & -25.1 & 3.3 \\
\hline $15 X-2,117-147$ & 180.7 & 0.66 & 0.06 & 0.12 & 0.20 & 0.81 & 430 & 0.44 & 45 & 184 & 7.0 & 3.8 & -27.2 & 1.9 \\
\hline $15 X-4,115-150$ & 183.7 & 0.45 & 0.05 & 0.04 & 0.18 & 0.54 & 428 & 0.29 & 62 & 186 & 5.7 & 6.9 & -27.7 & 5.2 \\
\hline $16 \mathrm{X}-2,120-150$ & 190.4 & 2.54 & 0.15 & 0.80 & 0.87 & 2.49 & 416 & 1.31 & 66 & 190 & 9.0 & 1.6 & -25.7 & 5.1 \\
\hline $16 X-4,21-28$ & 192.2 & 0.60 & 0.04 & 0.50 & 0.11 & 0.57 & 414 & 0.26 & 42 & 219 & 5.9 & 0.5 & -25.9 & 6.2 \\
\hline $16 X-4,28-39$ & 192.3 & 1.70 & 0.08 & 0.50 & 0.26 & 1.25 & 413 & 0.67 & 39 & 187 & 8.4 & 1.3 & -26.5 & 5.0 \\
\hline $17 X-2,120-150$ & 200.0 & 1.18 & 0.08 & 0.11 & 0.24 & 1.23 & 417 & 0.50 & 48 & 246 & 6.7 & 4.5 & -26.3 & 1.8 \\
\hline $17 X-3,120-150$ & 201.5 & 1.46 & 0.07 & 0.10 & 0.24 & 1.13 & 421 & 0.46 & 52 & 246 & 6.2 & 4.7 & -24.8 & 2.6 \\
\hline $18 X-1,120-130$ & 208.0 & 0.87 & 0.05 & 0.33 & 0.16 & 0.80 & 422 & 0.50 & 32 & 160 & 10.2 & 1.5 & -26.2 & 5.4 \\
\hline
\end{tabular}


Table T1 (continued).

\begin{tabular}{|c|c|c|c|c|c|c|c|c|c|c|c|c|c|c|}
\hline \multirow{3}{*}{$\begin{array}{l}\text { Core, section, } \\
\text { interval }(\mathrm{cm})\end{array}$} & \multirow{3}{*}{$\begin{array}{l}\text { Depth } \\
\text { (mbsf) }\end{array}$} & \multirow{2}{*}{\multicolumn{3}{|c|}{ Major elements (wt\%) }} & \multicolumn{8}{|c|}{ Rock-Eval pyrolysis } & & \\
\hline & & & & & \multirow{2}{*}{$\begin{array}{c}\mathrm{S}_{2} \\
\text { (mg HC/ } \\
\text { g rock) }\end{array}$} & \multirow{2}{*}{$\begin{array}{c}\mathrm{S}_{3} \\
\text { (mg rock) }\end{array}$} & \multirow{2}{*}{$\begin{array}{l}T_{\max } \\
\left({ }^{\circ} \mathrm{C}\right)\end{array}$} & \multirow{2}{*}{$\begin{array}{l}\text { TOC } \\
\text { (wt\%) }\end{array}$} & \multirow{2}{*}{$\begin{array}{c}\mathrm{HI} \\
\text { (mg HC/ } \\
\mathrm{g} \text { TOC) }\end{array}$} & \multirow{2}{*}{$\begin{array}{c}\mathrm{OI} \\
\left(\mathrm{mg} \mathrm{CO}_{2} /\right. \\
\mathrm{g} \mathrm{TOC})\end{array}$} & \multirow{2}{*}{$\begin{array}{l}\text { TOC/ } \\
\text { TN }\end{array}$} & \multirow{2}{*}{$\begin{array}{l}\text { TOC/ } \\
\text { TS }\end{array}$} & Isotope & $s(\%)$ \\
\hline & & TC & $\mathrm{TN}$ & TS & & & & & & & & & $\delta^{13} \mathrm{C}$ & $\delta^{15} \mathrm{~N}$ \\
\hline $18 X-2,0-25$ & 208.1 & 1.29 & 0.07 & 0.16 & 0.30 & 1.23 & 400 & 0.51 & 59 & 241 & 6.9 & 3.1 & -25.7 & 4.3 \\
\hline $18 X-3,67-72$ & 209.7 & 0.64 & 0.04 & 0.67 & 0.11 & 0.53 & 328 & 0.29 & 38 & 183 & 6.7 & 0.4 & -26.2 & 3.8 \\
\hline $18 X-3,72-82$ & 209.8 & 1.41 & 0.07 & 0.45 & 0.23 & 1.06 & 400 & 0.53 & 43 & 200 & 7.4 & 1.2 & -24.6 & 2.8 \\
\hline $19 X-1,20-40$ & 216.6 & 1.14 & 0.09 & 1.13 & 0.45 & 1.00 & 412 & 0.76 & 59 & 132 & 8.9 & 0.7 & -24.2 & 2.4 \\
\hline $19 X-3,120-150$ & 219.9 & 1.46 & 0.08 & 0.93 & 0.39 & 1.02 & 410 & 0.70 & 56 & 146 & 8.3 & 0.8 & -25.5 & 3.3 \\
\hline $20 X-1,120-150$ & 227.4 & 0.39 & 0.05 & 0.77 & 0.12 & 0.36 & 424 & 0.30 & 40 & 120 & 6.1 & 0.4 & -25.1 & -0.6 \\
\hline $20 X-4,120-150$ & 230.9 & 0.67 & 0.06 & 0.72 & 0.13 & 0.57 & 409 & 0.35 & 37 & 163 & 5.9 & 0.5 & -26.4 & 2.8 \\
\hline $21 \mathrm{X}-2,122-150$ & 238.2 & 0.55 & 0.05 & 0.65 & 0.11 & 0.44 & 420 & 0.39 & 28 & 113 & 8.5 & 0.6 & -26.5 & 5.2 \\
\hline $21 X-4,120-150$ & 241.2 & 0.72 & 0.07 & 0.46 & 0.19 & 0.60 & 422 & 0.51 & 37 & 118 & 7.4 & 1.1 & -26.4 & 3.5 \\
\hline $22 X-2,51-61$ & 247.3 & 0.59 & 0.06 & 0.82 & 0.18 & 0.51 & 395 & 0.36 & 50 & 142 & 5.6 & 0.4 & -24.3 & 3.0 \\
\hline $22 X-4,120-150$ & 250.2 & 1.34 & 0.08 & 0.63 & 0.29 & 0.96 & 411 & 0.61 & 48 & 157 & 7.6 & 1.0 & -25.9 & 4.2 \\
\hline $23 X-3,120-150$ & 259.2 & 0.68 & 0.05 & 0.50 & 0.11 & 0.61 & 424 & 0.29 & 38 & 210 & 5.9 & 0.6 & -26.7 & 4.5 \\
\hline $24 X-4,0-40$ & 268.9 & 0.48 & 0.06 & 0.12 & 0.14 & 0.42 & 414 & 0.38 & 37 & 111 & 6.9 & 3.1 & -26.0 & -1.8 \\
\hline $25 X-4,0-40$ & 278.8 & 0.67 & 0.06 & 0.18 & 0.17 & 0.57 & 429 & 0.39 & 44 & 146 & 6.6 & 2.2 & -25.5 & 4.0 \\
\hline $26 \mathrm{X}-4,0-30$ & 288.4 & 1.35 & 0.13 & 0.61 & 0.75 & 1.23 & 415 & 1.02 & 74 & 121 & 7.8 & 1.7 & -24.2 & 4.7 \\
\hline $27 X-4,0-50$ & 298.0 & 0.97 & 0.08 & 0.24 & 0.48 & 0.88 & 414 & 0.68 & 71 & 129 & 8.1 & 2.9 & -25.1 & 4.2 \\
\hline 311-U1329C- & & & & & & & & & & & & & & \\
\hline $1 \mathrm{H}-1,140-150$ & 1.5 & 0.82 & 0.07 & 0.25 & 0.25 & 0.64 & 426 & 0.49 & 51 & 131 & 7.5 & 2.0 & -27.2 & 2.1 \\
\hline $1 \mathrm{H}-3,140-150$ & 4.5 & 0.82 & 0.04 & 0.26 & 0.23 & 0.88 & 426 & 0.52 & 44 & 169 & 14.1 & 2.0 & -25.7 & 4.0 \\
\hline $1 \mathrm{H}-5,140-150$ & 7.5 & 1.35 & 0.07 & 0.16 & 0.40 & 1.34 & 412 & 0.58 & 69 & 231 & 7.8 & 3.7 & -24.8 & 5.1 \\
\hline $2 \mathrm{H}-1,140-150$ & 9.6 & 1.56 & 0.15 & 0.65 & 1.40 & 1.60 & 412 & 1.23 & 114 & 130 & 8.4 & 1.9 & -23.7 & 6.7 \\
\hline $2 \mathrm{H}-3,140-150$ & 12.6 & 1.23 & 0.07 & 0.20 & 0.34 & 1.15 & 406 & 0.62 & 55 & 185 & 8.4 & 3.1 & -24.4 & 5.6 \\
\hline $2 \mathrm{H}-5,140-150$ & 15.6 & 0.59 & 0.05 & 0.49 & 0.13 & 0.68 & 335 & 0.36 & 36 & 189 & 7.8 & 0.7 & -25.9 & 3.7 \\
\hline $2 \mathrm{H}-7,74-84$ & 17.9 & 2.76 & 0.07 & 0.69 & 0.23 & 1.38 & 385 & 0.56 & 41 & 246 & 8.6 & 0.8 & -24.9 & 5.1 \\
\hline $3 \mathrm{H}-2,135-150$ & 20.5 & 1.22 & 0.07 & 0.87 & 0.20 & 1.00 & 399 & 0.49 & 41 & 204 & 7.5 & 0.6 & -24.9 & 5.0 \\
\hline $3 \mathrm{H}-4,135-150$ & 23.5 & 0.98 & 0.06 & 1.01 & 0.15 & 0.72 & 342 & 0.43 & 35 & 167 & 7.5 & 0.4 & -25.8 & 4.4 \\
\hline $3 \mathrm{H}-6,135-150$ & 26.5 & 0.81 & 0.05 & 0.71 & 0.16 & 0.59 & 429 & 0.38 & 42 & 155 & 7.9 & 0.5 & -26.4 & 4.2 \\
\hline $4 \mathrm{H}-2,120-150$ & 30.0 & 1.22 & 0.07 & 1.00 & 0.32 & 1.12 & 392 & 0.62 & 52 & 181 & 8.9 & 0.6 & -25.0 & 4.3 \\
\hline $4 \mathrm{H}-5,120-150$ & 34.5 & 1.35 & 0.08 & 0.88 & 0.47 & 1.22 & 402 & 0.83 & 57 & 147 & 10.6 & 0.9 & -25.0 & 4.8 \\
\hline $5 \mathrm{H}-2,130-150$ & 39.5 & 1.13 & 0.10 & 1.02 & 0.68 & 1.15 & 400 & 0.97 & 70 & 119 & 9.5 & 1.0 & -24.4 & 3.9 \\
\hline $5 \mathrm{H}-5,130-150$ & 44.0 & 0.99 & 0.06 & 1.61 & 0.22 & 0.76 & 408 & 0.45 & 49 & 169 & 8.0 & 0.3 & -25.3 & 3.4 \\
\hline $6 \mathrm{H}-1,135-150$ & 47.5 & 1.59 & 0.10 & 1.20 & 0.82 & 1.40 & 403 & 0.92 & 89 & 152 & 8.9 & 0.8 & -24.4 & 5.1 \\
\hline $6 \mathrm{H}-5,135-150$ & 53.5 & 0.54 & 0.04 & 0.55 & 0.20 & 0.37 & 422 & 0.42 & 48 & 88 & 9.8 & 0.8 & -26.7 & 4.0 \\
\hline $7 P-1,25-50$ & 56.0 & 1.33 & 0.09 & 1.07 & 0.87 & 1.26 & 406 & 0.92 & 95 & 137 & 10.0 & 0.9 & -24.6 & 4.8 \\
\hline $8 \mathrm{H}-2,135-150$ & 60.5 & 1.70 & 0.14 & 1.07 & 1.34 & 1.67 & 411 & 1.31 & 102 & 127 & 9.4 & 1.2 & -24.4 & 6.0 \\
\hline $8 \mathrm{H}-5,135-150$ & 65.0 & 0.95 & 0.06 & 0.51 & 0.21 & 0.90 & 420 & 0.40 & 52 & 225 & 6.9 & 0.8 & -25.8 & 3.2 \\
\hline $9 \mathrm{H}-2,135-150$ & 70.0 & 2.00 & 0.08 & 0.50 & 0.39 & 1.54 & 406 & 0.58 & 67 & 266 & 7.2 & 1.2 & -24.6 & 4.0 \\
\hline $9 \mathrm{H}-5,130-150$ & 74.5 & 2.29 & 0.11 & 0.86 & 0.80 & 1.71 & 410 & 0.93 & 86 & 184 & 8.7 & 1.1 & -24.2 & 3.7 \\
\hline $10 \mathrm{H}-2,130-150$ & 79.5 & 1.36 & 0.10 & 0.28 & 0.46 & 1.38 & 408 & 0.77 & 60 & 179 & 7.5 & 2.8 & -24.9 & 3.3 \\
\hline $10 \mathrm{H}-5,130-150$ & 84.0 & 1.59 & 0.11 & 1.11 & 0.65 & 1.27 & 416 & 0.96 & 68 & 132 & 9.1 & 0.9 & -24.4 & 4.1 \\
\hline $11 \mathrm{H}-2,130-150$ & 89.0 & 1.69 & 0.12 & 0.51 & 0.76 & 1.60 & 413 & 1.01 & 75 & 158 & 8.7 & 2.0 & -24.5 & 5.1 \\
\hline $11 \mathrm{H}-5,130-150$ & 93.5 & 0.64 & 0.05 & 0.26 & 0.18 & 0.60 & 425 & 0.40 & 45 & 150 & 7.4 & 1.5 & -26.5 & 4.6 \\
\hline $12 \mathrm{H}-2,120-150$ & 98.5 & 1.76 & 0.15 & 1.17 & 1.50 & 1.66 & 414 & 1.29 & 116 & 129 & 8.4 & 1.1 & -24.0 & 4.7 \\
\hline $12 \mathrm{H}-5,130-150$ & 103.0 & 1.37 & 0.11 & 0.81 & 0.61 & 1.43 & 416 & 0.92 & 66 & 155 & 8.7 & 1.1 & -24.8 & 4.4 \\
\hline $13 \mathrm{H}-2,130-150$ & 108.0 & 1.32 & 0.10 & 0.72 & 0.54 & 1.28 & 413 & 0.82 & 66 & 156 & 8.4 & 1.1 & -24.2 & 2.8 \\
\hline $13 \mathrm{H}-5,130-150$ & 112.5 & 1.11 & 0.11 & 0.45 & 0.48 & 1.10 & 418 & 0.74 & 65 & 149 & 6.9 & 1.7 & -24.1 & 4.0 \\
\hline $15 \mathrm{H}-2,130-150$ & 119.5 & 1.83 & 0.18 & 0.56 & 1.57 & 1.58 & 415 & 1.64 & 96 & 96 & 9.2 & 3.0 & -23.7 & 5.4 \\
\hline $15 \mathrm{H}-5,130-150$ & 124.0 & 1.74 & 0.18 & 0.63 & 1.56 & 1.47 & 416 & 1.59 & 98 & 92 & 8.6 & 2.5 & -24.2 & 5.1 \\
\hline $16 \mathrm{H}-2,130-150$ & 129.0 & 1.13 & 0.09 & 0.30 & 0.31 & 1.02 & 424 & 0.60 & 52 & 170 & 6.9 & 2.0 & -25.0 & 3.6 \\
\hline $16 \mathrm{H}-5,130-150$ & 133.5 & 0.79 & 0.06 & 0.92 & 0.15 & 0.75 & 422 & 0.34 & 44 & 221 & 5.6 & 0.4 & -25.9 & 2.8 \\
\hline $17 \mathrm{H}-2,125-145$ & 138.5 & 1.46 & 0.14 & 0.90 & 0.92 & 1.11 & 422 & 1.33 & 69 & 83 & 9.8 & 1.5 & -24.6 & 3.3 \\
\hline $17 \mathrm{H}-3,130-150$ & 140.0 & 1.29 & 0.13 & 0.86 & 0.69 & 1.04 & 416 & 1.13 & 61 & 92 & 8.5 & 1.3 & -25.1 & 3.8 \\
\hline $18 X-3,125-150$ & 144.6 & 0.97 & 0.09 & 0.66 & 0.29 & 0.55 & 411 & 0.65 & 45 & 85 & 7.1 & 1.0 & -25.4 & 3.1 \\
\hline $19 X-2,120-150$ & 152.8 & 1.51 & 0.14 & 0.33 & 0.80 & 1.15 & 421 & 1.05 & 76 & 110 & 7.6 & 3.2 & -24.1 & 4.2 \\
\hline $20 X-2,120-150$ & 162.5 & 1.63 & 0.17 & 1.38 & 1.44 & 1.08 & 411 & 1.41 & 102 & 77 & 8.3 & 1.0 & -23.0 & 3.8 \\
\hline $21 X-6,0-30$ & 176.5 & 0.79 & 0.07 & 0.23 & 0.23 & 0.55 & 430 & 0.51 & 45 & 108 & 7.5 & 2.2 & -25.5 & 4.1 \\
\hline $22 X-4,0-35$ & 183.6 & 1.25 & 0.10 & 0.31 & 0.53 & 1.08 & 421 & 0.82 & 65 & 132 & 8.0 & 2.6 & -24.4 & 4.3 \\
\hline
\end{tabular}

Note: ${ }^{*}=$ clay. 